

\title{
A CONSISTENT REFINEMENT OF FIRST-ORDER SHEAR DEFORMATION THEORY FOR LAMINATED COMPOSITE AND SANDWICH PLATES USING IMPROVED ZIGZAG KINEMATICS
}

\author{
Alexander Tessler, Marco Di Sciuva And Marco Gherlone
}

\begin{abstract}
A refined zigzag theory is presented for laminated-composite and sandwich plates that includes the kinematics of first-order shear deformation theory as its baseline. The theory is variationally consistent and is derived from the virtual work principle. Novel piecewise-linear zigzag functions are used, providing a more realistic representation of the deformation states of transverse shear-flexible plates than other similar theories. The formulation does not enforce full continuity of the transverse shear stresses across the plate's thickness, yet it is robust. Transverse shear correction factors are not required to yield accurate results. The theory avoids the shortcomings of earlier zigzag theories (such as shearforce inconsistency and difficulties in simulating clamped boundary conditions) which have limited their accuracy. This new theory requires only $C^{0}$-continuous kinematic approximations and is perfectly suited for developing computationally efficient finite elements. It should be useful for obtaining relatively efficient, accurate estimates of structural response, needed in designing high-performance load-bearing aerospace structures.
\end{abstract}

A list of symbols can be found on page 363.

\section{Introduction}

The high-performance and lightweight characteristics of advanced composite materials have spurred numerous applications of these materials in military and civilian aircraft, aerospace vehicles, and naval and civil structures. To realize the full potential of composite structures, further advances in structural design and analysis methods are necessary. In particular, development of cost-effective and reliable laminated-composite structures as the primary load-bearing components of a vehicle requires further advances in stress analysis and failure prediction methodologies.

A wide variety of modern civilian and military aircraft employ relatively thick laminated composites, with one hundred or more layers, in the primary load-bearing structures. Such structures can exhibit pronounced transverse shear deformation and, under certain conditions, design-critical thickness-stretch deformations. Fail-safe design of these structures requires accurate stress-analysis methods, particularly for regions of stress concentration. Computationally efficient analytical models based on beam, plate, and shell assumptions that account for transverse shear and thickness-stretch deformations have recently been addressed in [Tessler 1993; Cook and Tessler 1998; Barut et al. 2002]. To achieve accurate computational models, three-dimensional finite element analyses are often preferred over beam, plate, and shell models based on first-order shear deformation theories (FSDT). This preference is due to the

Keywords: first-order shear deformation plate theory, zigzag kinematics, laminated composite plates, sandwich plates, virtual work principle. 
latter having the tendency to underestimate the normal stresses, particularly in highly heterogeneous and thick composite and sandwich laminates [Reissner 1985; Librescu et al. 1987; Noor and Burton 1989; Liu and Li 1996]. For composite laminates with hundreds of layers, however, 3D modeling becomes prohibitively expensive, especially for nonlinear and progressive failure analyses. To realize improved response predictions based on beam, plate, and shell assumptions, numerous refined theories have been developed [Ambartsumyan 1961; Sun and Whitney 1973; Lo et al. 1977a; 1977b; Reddy 1997]. Many of these theories have significant flaws in their theoretical foundation and predictive capabilities and, for these reasons, have not found general acceptance in practical applications.

One class of refined theories that has emerged as practical for engineering applications is known as zigzag theories [Di Sciuva 1984a; 1984b; 1985a; 1985b; 1986; 1987; 1990; 1992; Murakami 1986; Toledano and Murakami 1987; Cho and Parmerter 1992; 1993; Di Sciuva et al. 2002]. This class of theories employs a zigzag-like distribution for the in-plane displacements through the laminate thickness, while ensuring a fixed number of kinematic variables regardless of the number of material layers (or laminae). Using through-the-thickness linear in-plane kinematics with displacements and transverse shearing angles as primary variables, [Di Sciuva 1984a; 1984b; 1985a; 1985b; 1986; 1987] added a piecewise linear distribution known as the zigzag displacement. By explicitly enforcing a set of equilibrium conditions along lamina interfaces, constant transverse shear stresses are developed across the laminate thickness. These theories are often referred to as linear zigzag theories (or models) to delineate throughthe-thickness linear distributions of their baseline in-plane displacements. Di Sciuva [1990; 1992] and Cho and Parmerter [1992; 1993] provided further enhancements to the zigzag theory by adding quadratic and cubic power-series terms to the in-plane displacements. As in the previous efforts, the procedure relies on the transverse shear stress equilibrium constraints. The resulting transverse shear stresses are continuous across the laminate thickness, and vanish along the top and bottom laminate surfaces (see also [Di Sciuva et al. 2002]). Murakami and coworkers developed another linear zigzag theory [Murakami 1986] and a higher-order zigzag theory [Toledano and Murakami 1987] that also includes transverse normal deformations. The governing equations are derived using Reissner's mixed variational theorem [Reissner 1984], and they include transverse shear and transverse normal stresses [Toledano and Murakami 1987] as their primary unknowns.

Zigzag theories provide sufficiently accurate response predictions for relatively thick laminatedcomposite and sandwich structures including those for normal strains and stresses [Di Sciuva 1984a; 1984b; 1985a; 1985b; 1986; 1987; 1990; 1992; Murakami 1986; Toledano and Murakami 1987; Cho and Parmerter 1992; 1993; Di Sciuva et al. 2002]. Furthermore, these theories often yield response predictions comparable to those of layer-wise and higher-order theories that are more computationally intensive. To make a zigzag theory practical for large-scale analyses and engineering design, its analytic framework must be well suited for an efficient finite element approximation. While pursuing a computationally desirable zigzag beam theory, Averill [1994; Averill and Yip 1996] recognized two significant drawbacks that plague many previously mentioned zigzag theories, in particular those having displacements as primary unknowns [Di Sciuva 1984a; 1984b; 1985a; 1985b; 1986; 1987; 1990; 1992; Cho and Parmerter 1992; 1993]: Firstly, because the curvature is expressed as a second spatial derivative of the deflection variable, $C^{1}$ continuous functions are required to approximate the deflection within the finite element framework. Due to their complexity and overly stiff response properties, such approximations are especially undesirable for plate and shell finite elements. Secondly, transverse shear 
stresses calculated from constitutive equations vanish erroneously along the clamped boundaries, this being a "physical inconsistency that plagues many popular shear deformation theories" [Averill 1994]. Averill resolved the first issue by adopting the kinematics of Timoshenko's shear deformation theory as a baseline; however, to resolve the second issue he proposed a boundary-condition compromise at the expense of the variational consistency of the theory, in which a kinematic variable representing the amplitude of the zigzag displacement is omitted from the variationally required set of boundary conditions.

Tessler et al. [2007] presented a clearer insight into the existing flaws of the linear zigzag models [Di Sciuva 1984a; 1984b; 1985a; 1985b; 1986; 1987]. In these earlier theories, the kinematic field is constructed by way of enforcing equilibrium of the transverse shear stresses along lamina interfaces, which results in constant (uniform) transverse shear stresses across the laminate thickness. The consequence of the transverse shear equilibrium constraints within a displacement-based theory is that they tend to overconstrain the kinematic field. As delineated in [Tessler et al. 2007], the overconstraint is manifested by a physical inconsistency (or anomaly) in the definition of the transverse shear forces. Consequently, the cross-sectional integral of the transverse shear stress does not correspond to the transverse shear force obtained from the bending equilibrium equation of the theory. A further byproduct of this anomaly is the erroneous vanishing of the transverse shear strains, stresses, and forces along the clamped boundaries. Averill [1994] pointed out the latter aspect; however, as previously discussed, he proposed only a variationally inconsistent strategy to circumvent the issue. It is also worth mentioning that the same transverse shear stress anomaly exists in many other theories, for example, the cubic zigzag theories by Di Sciuva [1990; 1992] and Cho and Parmerter [1992; 1993], and the smeared cubic theory by Reddy [1984; 1997; 2004]. Also note that almost exclusively, and this pertains to all of the aforementioned articles, the proposed theories are assessed by solving example problems with simply supported boundaries, where the solutions inherently do not exhibit transverse shear anomalies. Regrettably, the few contributions that considered clamped boundary conditions, for example [Averill 1994; Umasree and Bhaskar 2006], do not discuss shear stress distributions along the clamped boundary.

$\mathrm{Yu}$ [2005] proposed a variational asymptotic plate and shell analysis (VAPAS), and Kim and Cho [2005; 2006] presented an enhanced first-order shear deformation theory (EFSDT). Both of these modeling strategies use a three-step procedure [Yu et al. 2008]: (1) obtain a 2D Reissner-Mindlin type constitutive model, (2) carry out the global plate analysis using the constitutive model from step (1), and (3) recover through-the-thickness distributions of displacements and stresses from step (1). In the framework of EFSDT, the recovery of through-the-thickness distributions is performed by means of the cubic zigzag theory by Cho and Parmerter [1992; 1993]. A critical review and comparison of the two approaches in [Yu et al. 2008] reveal that VAPAS requires high-order displacement derivatives to achieve adequate predictions for the in-plane and transverse normal stresses, whereas EFSDT is somewhat inaccurate in terms of the in-plane normal stresses and its extension to nonlinear problems may not be straightforward. Furthermore, since the recovery of the 3D distributions is performed by means of the cubic zigzag theory by Cho and Parmerter [1992; 1993], it is argued that EFSDT should be affected by the aforementioned transverse shear stress anomaly. Unfortunately, Kim and Cho [2005; 2006] discuss numerical results only for plates with simply supported boundary conditions.

To construct a computationally attractive theory suitable for FEM without the flaws of the previous zigzag models, Tessler et al. [2007] proposed a refined zigzag theory for laminated composite and 
sandwich beams. They derived a novel zigzag function without enforcing a full transverse shear stress equilibrium along the lamina interfaces. The shear force definitions are fully consistent with respect to the physical and variational requirements. The resulting theory is without the aforementioned flaws of the previous zigzag theories and has been shown to demonstrate consistently superior results.

A successful extension of this new zigzag methodology to plate theory was advanced in [Tessler et al. 2009]; in the present we detail the theoretical foundation of the refined zigzag plate theory introduced there. The theory's applicability to laminated composite and sandwich plates is demonstrated by presenting example problems with simply supported and clamped boundaries and which include relatively thick laminates with a high degree of transverse shear flexibility, anisotropy, and heterogeneity. This new formulation augments FSDT with an improved zigzag kinematic field that involves a novel $C^{0}$ continuous (across lamina interfaces) representation of the in-plane displacements. The kinematic field is independent of the number of material layers and does not require enforcement of transverse shear stress continuity to yield accurate results. Unlike other similar theories [Di Sciuva 1984a; Averill 1994], the zigzag contribution to the in-plane displacement field is physically realistic, is zero-valued at the top and bottom plate surfaces, and accounts for the shear deformation of every lamina in a consistent way. As a result, transverse shear correction factors are not needed. Additionally, the plate equilibrium equations, constitutive equations, boundary conditions, and strain-displacement relations are consistently derived from the virtual work principle. Moreover, the analytical form of this new theory is ideally suited for developing computationally efficient finite elements requiring only $C^{0}$-continuity. This benefit will enable an efficient use of accurate zigzag approximations in large-scale analyses to facilitate the development of robust designs of high-performance aerospace vehicles.

In the remainder of the paper, the theoretical foundation of the new theory and its quantitative assessment are detailed. The zigzag kinematic assumptions, strain-displacement equations, and constitutive lamina relations are presented in Section 2. A set of unique zigzag functions is then introduced and their mathematical structure is described in Section 3. The plate equilibrium equations and their associated boundary conditions, derived from the virtual work principle, are presented in Section 4. In Section 5, an extensive quantitative assessment of the theory is carried out, using closed-form solutions for simply supported and cantilevered plates made of laminated-composite and sandwich material systems. Some of the example problems represent significant challenges for any approximate theory. It is demonstrated that this new zigzag theory eliminates a major flaw of other similar theories; that is, the theory enables accurate modeling of the clamped boundary condition while adhering strictly to the variationally required boundary conditions. Finally, in Section 6, several concluding remarks emphasizing the merits of the new theory are highlighted.

\section{Kinematics and formulation}

Consider a laminated plate of uniform thickness $2 h$ with $N$ perfectly bonded orthotropic layers (or laminae) as shown in Figure 1. Points of the plate are located by the orthogonal Cartesian coordinates $\left(x_{1}, x_{2}, z\right)$. The ordered pair $\left(x_{1}, x_{2}\right) \in S_{m}$ denotes the in-plane coordinates, where $S_{m}$ represents the set of points given by the intersection of the plate with the plane $z=0$, referred to herein as the middle reference plane (or mid-plane). The symbol $z \in[-h, h]$ denotes the through-the-thickness coordinate, with $z=0$ identifying the plate's mid-plane. The plate is subjected to a normal-pressure loading, $q\left(x_{1}, x_{2}\right)$, 

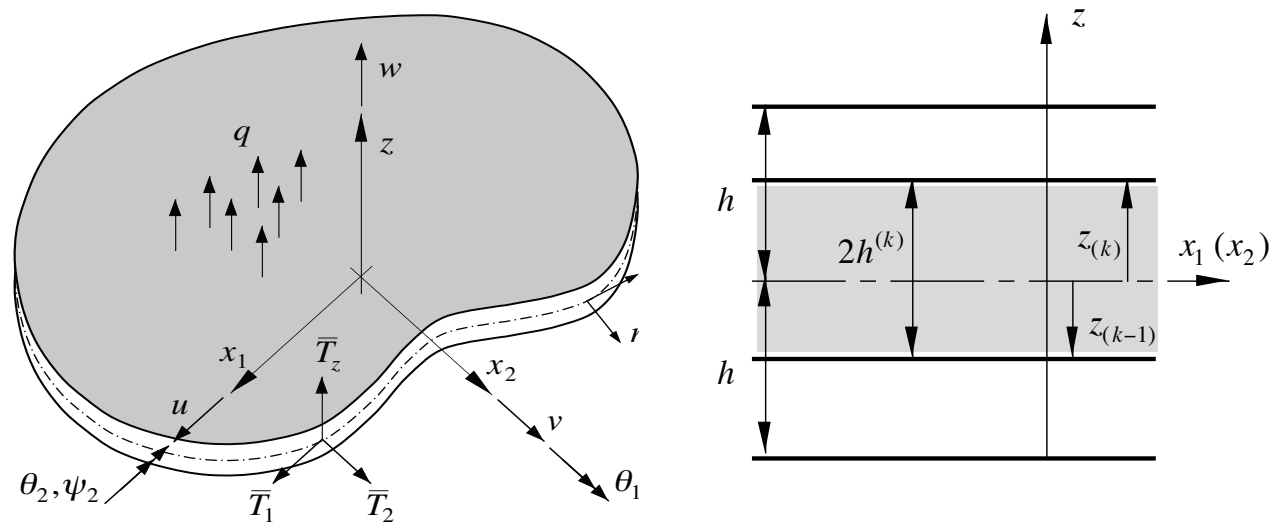

Figure 1. General plate notation (left), and lamination notation (right).

attributed to the mid-plane, $S_{m}$, that is defined as positive in the positive $z$ direction. In addition, a traction vector, $\left(\bar{T}_{1}, \bar{T}_{2}, \bar{T}_{z}\right)$, is prescribed on $S_{\sigma} \subset S$, where $S$ denotes the total cylindrical-edge surface. On the remaining part of the edge surface, $S_{u} \subset S$, displacement restraints are imposed (or prescribed). The sections of the plate edge are related by $S_{\sigma} \cup S_{u}=S$ and $S_{\sigma} \cap S_{u}=\varnothing$. Moreover, the curves $C_{\sigma}=S_{\sigma} \cap S_{m}$ and $C_{u}=S_{u} \cap S_{m}$ define the two parts of the total perimeter $C=C_{\sigma} \cup C_{u}$ surrounding the mid-plane region, $S_{m}$. Finally, it is presumed that the constitutive properties may differ appreciably from lamina to lamina, the plate deformations result in small strains, and that body and inertial forces are negligible.

The orthogonal components of the displacement vector, corresponding to material points of the plate (or laminate), are expressed as

$$
\begin{aligned}
u_{1}^{(k)}\left(x_{1}, x_{2}, z\right) & \equiv u\left(x_{1}, x_{2}\right)+z \theta_{1}\left(x_{1}, x_{2}\right)+\phi_{1}^{(k)}(z) \psi_{1}\left(x_{1}, x_{2}\right), \\
u_{2}^{(k)}\left(x_{1}, x_{2}, z\right) & \equiv v\left(x_{1}, x_{2}\right)+z \theta_{2}\left(x_{1}, x_{2}\right)+\phi_{2}^{(k)}(z) \psi_{2}\left(x_{1}, x_{2}\right), \\
u_{z}\left(x_{1}, x_{2}, z\right) & \equiv w\left(x_{1}, x_{2}\right)
\end{aligned}
$$

where the in-plane displacement components $u_{1}^{(k)}$ and $u_{2}^{(k)}$ are comprised of constant, linear, and zigzag variations through the thickness. The zigzag variations are $C_{0}$-continuous functions with discontinuous thickness-direction derivatives along the lamina interfaces. The superscript $(k)$ is used to indicate quantities corresponding to the $k$-th lamina, whereas the subscript $(k)$ defines quantities corresponding to the interface between the $k$-th and $(k+1)$-th laminae. Thus, the $k$-th lamina thickness is defined in the range $z \in\left[z_{(k-1)}, z_{(k)}\right](k=1, \ldots, N)$; see Figure 1 . The transverse displacement $u_{z}$ is assumed to be constant through the thickness and is independent of constitutive properties of the $k$-th lamina; hence the superscript $(k)$ does not appear in its definition.

The kinematic variables in (1) can be interpreted as follows. For homogeneous plates, the zigzag functions $\phi_{\alpha}^{(k)}(\alpha=1,2)$ vanish identically and (1) yield the kinematics of FSDT. For this degenerate case, $u$ and $v$ represent the mid-plane displacements along the coordinate directions $x_{1}$ and $x_{2}$, respectively; $\theta_{1}$ and $\theta_{2}$ represent average bending rotations of the transverse normal about the positive $x_{2}$ and the negative $x_{1}$ directions, respectively; and $w$ is the transverse deflection. For more precise definitions of the kinematic variables within FSDT refer, for example, to [Tessler 1993]. The symbols $\phi_{\alpha}^{(k)}(\alpha=1,2)$ 
denote through-the-thickness piecewise-linear zigzag functions associated with heterogeneous plates, yet to be defined. The $\psi_{\alpha}=\psi_{\alpha}\left(x_{1}, x_{2}\right)(\alpha=1,2)$ functions represent the spatial amplitudes of the zigzag displacements and, together with the other five kinematic variables, are the unknowns in the analysis. The zigzag displacements $\phi_{\alpha}^{(k)} \psi_{\alpha}(\alpha=1,2)$ may be regarded as corrections to the in-plane displacements associated with laminate heterogeneity.

Consistent with the kinematic assumptions in (1), the theory accounts for transverse shear deformation. (Transverse normal deformations are neglected in the kinematics; however, their inclusion may be possible following, for example, [Tessler 1993]). Correspondingly, the in-plane and transverse shear strains are

$$
\begin{aligned}
& \varepsilon_{11}^{(k)}=u_{, 1}+z \theta_{1,1}+\phi_{1}^{(k)} \psi_{1,1}, \\
& \varepsilon_{22}^{(k)}=v_{, 2}+z \theta_{2,2}+\phi_{2}^{(k)} \psi_{2,2}, \\
& \gamma_{12}^{(k)}=u_{, 2}+v_{, 1}+z\left(\theta_{1,2}+\theta_{2,1}\right)+\phi_{1}^{(k)} \psi_{1,2}+\phi_{2}^{(k)} \psi_{2,1}, \\
& \gamma_{\alpha z}^{(k)}=\gamma_{\alpha}+\beta_{\alpha}^{(k)} \psi_{\alpha} \quad(\alpha=1,2),
\end{aligned}
$$

where, henceforward, $(\bullet)_{, \alpha} \equiv \frac{\partial(\bullet)}{\partial x_{\alpha}}$ denotes a partial derivative with respect to the mid-plane coordinate, $x_{\alpha}(\alpha=1,2)$. Also, the following notation is introduced

$$
\begin{aligned}
\gamma_{\alpha} \equiv w_{, \alpha}+\theta_{\alpha} & (\alpha=1,2), \\
\beta_{\alpha}^{(k)} \equiv \frac{\partial}{\partial z}\left(\phi_{\alpha}^{(k)}\right) & (\alpha=1,2),
\end{aligned}
$$

where the shear angles $\gamma_{\alpha}$ are uniform through the total laminate thickness, and $\beta_{\alpha}^{(k)}$ are piecewise constant functions that are uniform through the thickness of each individual lamina.

The generalized Hooke's law for the $k$-th orthotropic lamina, whose principal material directions are arbitrary with respect to the mid-plane reference coordinates, $\left(x_{1}, x_{2}\right) \in S_{m}$, is written as

$$
\left\{\begin{array}{l}
\sigma_{11} \\
\sigma_{22} \\
\tau_{12} \\
\tau_{2 z} \\
\tau_{1 z}
\end{array}\right\}^{(k)}=\left[\begin{array}{ccccc}
C_{11} & C_{12} & C_{16} & 0 & 0 \\
C_{12} & C_{22} & C_{26} & 0 & 0 \\
C_{16} & C_{26} & C_{66} & 0 & 0 \\
0 & 0 & 0 & Q_{22} & Q_{12} \\
0 & 0 & 0 & Q_{12} & Q_{11}
\end{array}\right]^{(k)}\left\{\begin{array}{l}
\varepsilon_{11} \\
\varepsilon_{22} \\
\gamma_{12} \\
\gamma_{2 z} \\
\gamma_{1 z}
\end{array}\right\},
$$

where $C_{i j}^{(k)}(i, j=1,2,6)$ and $Q_{p q}^{(k)}(p, q=1,2)$ are the transformed elastic stiffness coefficients referred to the $\left(x_{1}, x_{2}, z\right)$ coordinate system and relative to the plane-stress condition that ignores the transversenormal stress. The expressions for these coefficients in terms of the elastic moduli corresponding to the material coordinates can be found, for example, in [Reddy 1997].

\section{Refined zigzag functions and transverse shear constitutive relations}

The refined zigzag functions (or zigzag displacements) of the present theory are defined by piecewise linear, $C^{0}$-continuous functions through the laminate thickness. For convenience, the zigzag functions $\phi_{1}^{(k)}$ and $\phi_{2}^{(k)}$, which have units of length, are defined in terms of their respective lamina-interface values $u_{(i)}$ and $v_{(i)}(i=0,1, \ldots, N)$ (see Figure 2 depicting the notation for a three-layered laminate). 
Thus, for the $k$-th lamina located in the range $\left[z_{(k-1)}, z_{(k)}\right], \phi_{1}^{(k)}$ and $\phi_{2}^{(k)}$ are given as

$$
\phi_{1}^{(k)} \equiv \frac{1}{2}\left(1-\xi^{(k)}\right) u_{(k-1)}+\frac{1}{2}\left(1+\xi^{(k)}\right) u_{(k)}, \quad \phi_{2}^{(k)} \equiv \frac{1}{2}\left(1-\xi^{(k)}\right) v_{(k-1)}+\frac{1}{2}\left(1+\xi^{(k)}\right) v_{(k)},
$$

where

$$
\xi^{(k)}=\left[\frac{z-z_{(k-1)}}{h^{(k)}}-1\right] \in[-1,1] \quad(k=1, \ldots, N),
$$

with the first lamina beginning at $z_{(0)}=-h$, the last $\left(N\right.$-th) lamina ending at $z_{(N)}=h$, and the $k$-th lamina ending at $z_{(k)}=z_{(k-1)}+2 h^{(k)}$, where $2 h^{(k)}$ denotes the $k$-th lamina thickness.

Evaluating (4) at the laminae interfaces gives rise to the definitions of the interfacial displacements

$$
\begin{array}{ll}
u_{(k-1)}=\phi_{1}^{(k)}\left(\xi^{(k)}=-1\right), & u_{(k)}=\phi_{1}^{(k)}\left(\xi^{(k)}=1\right), \\
v_{(k-1)}=\phi_{2}^{(k)}\left(\xi^{(k)}=-1\right), & v_{(k)}=\phi_{2}^{(k)}\left(\xi^{(k)}=1\right) \quad(k=1, \ldots, N),
\end{array}
$$

where the interfacial displacements at the bottom and top plate surfaces are set herein to vanish identically, that is,

$$
u_{(0)}=u_{(N)}=v_{(0)}=v_{(N)}=0 .
$$

Substituting (4) into (2f) results in the piecewise constant functions $\beta_{\alpha}^{(k)}$ given by

$$
\left\{\begin{array}{c}
\beta_{1}^{(k)} \\
\beta_{2}^{(k)}
\end{array}\right\}=\frac{1}{2 h^{(k)}}\left\{\begin{array}{c}
u_{(k)}-u_{(k-1)} \\
v_{(k)}-v_{(k-1)}
\end{array}\right\} .
$$

Because the zigzag functions are zero-valued on the top and bottom surfaces, as defined by Equations (6a) and (6b), through-the-thickness integrals of the slope functions $\beta_{\alpha}^{(k)}(\alpha=1,2)$ vanish identically, that is,

$$
\int_{-h}^{h}\left\{\begin{array}{c}
\beta_{1}^{(k)} \\
\beta_{2}^{(k)}
\end{array}\right\} d z=\left\{\begin{array}{c}
\sum_{k=1}^{N} 2 h^{(k)} \beta_{1}^{(k)} \\
\sum_{k=1}^{N} 2 h^{(k)} \beta_{2}^{(k)}
\end{array}\right\}=\left\{\begin{array}{l}
0 \\
0
\end{array}\right\} .
$$
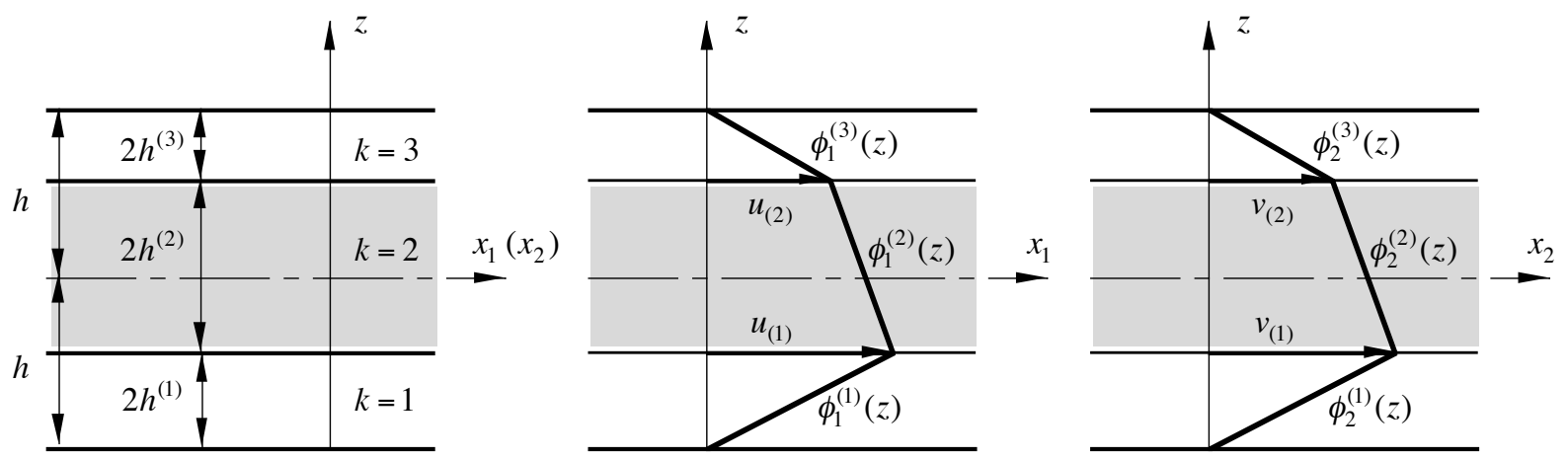

Figure 2. Notation for a three-layered laminate and $\phi_{1}^{(k)}$ and $\phi_{2}^{(k)}$ zigzag functions defined in terms of interfacial values (displacements), $u_{(i)}$ and $v_{(i)}(i=0,1, \ldots, N)$. 
Integrating (2d) across the laminate thickness and normalizing the result by the total laminate thickness reveals that

$$
\left\{\begin{array}{l}
\gamma_{1} \\
\gamma_{2}
\end{array}\right\}=\frac{1}{2 h} \int_{-h}^{h}\left\{\begin{array}{l}
\gamma_{1 z}^{(k)} \\
\gamma_{2 z}^{(k)}
\end{array}\right\} d z
$$

Thus, $\gamma_{\alpha}(\alpha=1,2)$ represent the average transverse shear strains, coinciding with the common representation of the transverse shear strains used in FSDT. Also, (9) indicates that the zigzag amplitude variables, $\psi_{\alpha}(\alpha=1,2)$, do not contribute to the average transverse shear strains.

The $u_{(k)}$ and $v_{(k)}$ interfacial values of the zigzag functions are obtained from (7) in terms of $\beta_{\alpha}^{(k)}$ $(\alpha=1,2 ; k=1, \ldots, N)$, that is,

$$
\left\{\begin{array}{l}
u_{(k)} \\
v_{(k)}
\end{array}\right\}=2 h^{(k)}\left\{\begin{array}{l}
\beta_{1}^{(k)} \\
\beta_{2}^{(k)}
\end{array}\right\}+\left\{\begin{array}{l}
u_{(k-1)} \\
v_{(k-1)}
\end{array}\right\} \quad(k=1, \ldots, N),
$$

or, alternatively,

$$
\left\{\begin{array}{l}
u_{(k)} \\
v_{(k)}
\end{array}\right\}=\left\{\begin{array}{l}
\sum_{i=1}^{k} 2 h^{(i)} \beta_{1}^{(i)} \\
\sum_{i=1}^{k} 2 h^{(i)} \beta_{2}^{(i)}
\end{array}\right\} \quad(k=1, \ldots, N) .
$$

Following the approach in [Tessler et al. 2007], the $\beta_{\alpha}^{(k)}$ functions are determined by first casting the transverse shear strains, $(2 \mathrm{~d})$, in terms of the transverse shear strain measures, $\eta_{\alpha} \equiv \gamma_{\alpha}-\psi_{\alpha}(\alpha=1,2)$, and the zigzag amplitude functions, $\psi_{\alpha}(\alpha=1,2)$, as

$$
\left\{\begin{array}{l}
\gamma_{1 z} \\
\gamma_{2 z}
\end{array}\right\}^{(k)} \equiv\left\{\begin{array}{l}
\eta_{1} \\
\eta_{2}
\end{array}\right\}+\left[\begin{array}{cc}
1+\beta_{1}^{(k)} & 0 \\
0 & 1+\beta_{2}^{(k)}
\end{array}\right]\left\{\begin{array}{l}
\psi_{1} \\
\psi_{2}
\end{array}\right\}
$$

The $\eta_{\alpha}$ strain measures are set to vanish explicitly in the theory of Di Sciuva [1984a] and enforced to vanish by way of penalty constraints in the theory of Averill [1994], thus equating $\gamma_{\alpha}$ to $\psi_{\alpha}$. Presently, no such constraints are imposed on these strain measures.

The transverse shear stresses using Equations (3) and (11) are given as

$$
\left\{\begin{array}{l}
\tau_{1 z} \\
\tau_{2 z}
\end{array}\right\}^{(k)} \equiv\left[\begin{array}{ll}
Q_{11} & Q_{12} \\
Q_{21} & Q_{22}
\end{array}\right]^{(k)}\left(\left\{\begin{array}{l}
\eta_{1} \\
\eta_{2}
\end{array}\right\}+\left[\begin{array}{cc}
1+\beta_{1}^{(k)} & 0 \\
0 & 1+\beta_{2}^{(k)}
\end{array}\right]\left\{\begin{array}{l}
\psi_{1} \\
\psi_{2}
\end{array}\right\}\right),
$$

or, alternatively, they can be expressed as

$$
\left\{\begin{array}{l}
\tau_{1 z} \\
\tau_{2 z}
\end{array}\right\}^{(k)} \equiv\left[\begin{array}{ll}
Q_{11} & Q_{12} \\
Q_{21} & Q_{22}
\end{array}\right]^{(k)}\left\{\begin{array}{l}
\eta_{1} \\
\eta_{2}
\end{array}\right\}+Q_{11}^{(k)}\left(1+\beta_{1}^{(k)}\right)\left\{\begin{array}{c}
1 \\
Q_{12}^{(k)} / Q_{11}^{(k)}
\end{array}\right\} \psi_{1}+Q_{22}^{(k)}\left(1+\beta_{2}^{(k)}\right)\left\{\begin{array}{c}
Q_{12}^{(k)} / Q_{22}^{(k)} \\
1
\end{array}\right\} \psi_{2} .
$$

In this form of the transverse shear constitutive relations, the stress vector associated with the $\eta_{\alpha}$ strain measures is independent of the zigzag functions. The second and third stress vectors include, as their normalization factors, the coefficients $Q_{\alpha \alpha}^{(k)}\left(1+\beta_{\alpha}^{(k)}\right)(\alpha=1,2)$ that are dependent on the zigzag functions through $\beta_{\alpha}^{(k)}$. Herein, these normalization factors are set to be constant quantities, denoted as $G_{\alpha}(\alpha=1,2)$, thus imposing constraint conditions on the distribution of the zigzag functions. 
These constraints give rise to the expressions

$$
\left\{\begin{array}{c}
\beta_{1}^{(k)} \\
\beta_{2}^{(k)}
\end{array}\right\}=\left\{\begin{array}{c}
G_{1} / Q_{11}^{(k)}-1 \\
G_{2} / Q_{22}^{(k)}-1
\end{array}\right\}
$$

The $G_{1}$ and $G_{2}$ constants are obtained by integrating (12b) through the laminate thickness while making use of (8), resulting in

$$
\left\{\begin{array}{l}
G_{1} \\
G_{2}
\end{array}\right\} \equiv\left\{\begin{array}{l}
\left(\frac{1}{2 h} \int_{-h}^{h} \frac{d z}{Q_{11}^{(k)}}\right)^{-1} \\
\left(\frac{1}{2 h} \int_{-h}^{h} \frac{d z}{Q_{22}^{(k)}}\right)^{-1}
\end{array}\right\}=\left\{\begin{array}{l}
\left(\frac{1}{h} \sum_{k=1}^{N} \frac{h^{(k)}}{Q_{11}^{(k)}}\right)^{-1} \\
\left(\frac{1}{h} \sum_{k=1}^{N} \frac{h^{(k)}}{Q_{22}^{(k)}}\right)^{-1}
\end{array}\right\},
$$

where it is seen that $G_{1}$ and $G_{2}$ are weighted-average transverse shear stiffness coefficients of their respective lamina-level coefficients, $Q_{11}^{(k)}$ and $Q_{22}^{(k)}$.

Substituting (12b) into (12a) results in the transverse shear constitutive relations of the form

$$
\left\{\begin{array}{l}
\tau_{1 z} \\
\tau_{2 z}
\end{array}\right\}^{(k)} \equiv\left[\begin{array}{ll}
Q_{11} & Q_{12} \\
Q_{21} & Q_{22}
\end{array}\right]^{(k)}\left\{\begin{array}{l}
\gamma_{1}+\psi_{1}\left(G_{1} / Q_{11}^{(k)}-1\right) \\
\gamma_{2}+\psi_{2}\left(G_{2} / Q_{22}^{(k)}-1\right)
\end{array}\right\},
$$

where, with the use of (12c), the dimensionless stiffness ratios are given as

$$
\frac{G_{\alpha}}{G_{\alpha \alpha}^{(k)}}=\left(\frac{Q_{\alpha \alpha}^{(k)}}{2 h} \int_{-h}^{h} \frac{d z}{Q_{\alpha \alpha}^{(k)}}\right)^{-1} \quad(\alpha=1,2) .
$$

These ratios are, in general, piecewise constant through the laminate thickness; however, for homogeneous plates, they are unit-valued. Thus, for homogeneous plates, the zigzag transverse shear contributions vanish, in which case (13a) becomes identical to the corresponding relations of FSDT.

The $\phi_{\alpha}^{(k)}(\alpha=1,2)$ zigzag functions are determined by substituting (10b) and (12b) into (4), while making use of (5), resulting in

$$
\begin{aligned}
\phi_{\alpha}^{(1)} & =(z+h)\left(\frac{G_{\alpha}}{Q_{\alpha \alpha}^{(1)}}-1\right) & (k=1), \\
\phi_{\alpha}^{(k)} & =(z+h)\left(\frac{G_{\alpha}}{Q_{\alpha \alpha}^{(k)}}-1\right)+\sum_{i=2}^{k} 2 h^{(i-1)}\left(\frac{G_{\alpha}}{Q_{\alpha \alpha}^{(i-1)}}-\frac{G_{\alpha}}{Q_{\alpha \alpha}^{(k)}}\right) & (k=2, \ldots, N), \\
z & \in\left[z_{(k-1)}, z_{(k)}\right], \quad z_{(0)}=-h, \quad z_{(k)}=z_{(k-1)}+2 h^{(k)} & (k=1, \ldots, N ; \alpha=1,2) .
\end{aligned}
$$

It is seen that the zigzag functions are independent of the state of deformation and are represented by $C^{0}$-continuous, piecewise linear functions of the thickness coordinate. The zigzag amplitudes, $\psi_{\alpha}$ $(\alpha=1,2)$, are vector functions of the actual response due to the applied loading, and they provide the proper scaling of the zigzag functions, thus controlling the total zigzag contribution to the in-plane displacements. The two zigzag amplitude functions and the remaining five kinematic variables constitute a set of seven kinematic variables associated with this refined zigzag plate theory. 


\section{Equilibrium equations, boundary conditions, and constitutive relations}

The plate equilibrium equations and boundary conditions are derived from the virtual work principle which, neglecting body forces and assuming zero shear tractions on the top and bottom bounding plate surfaces, may be written as

$$
\begin{aligned}
\int_{S_{m}} \int_{-h}^{h}\left(\sigma_{11}^{(k)} \delta \varepsilon_{11}^{(k)}+\sigma_{22}^{(k)} \delta \varepsilon_{22}^{(k)}+\right. & \left.\tau_{12}^{(k)} \delta \gamma_{12}^{(k)}+\tau_{1 z}^{(k)} \delta \gamma_{1 z}^{(k)}+\tau_{2 z}^{(k)} \delta \gamma_{2 z}^{(k)}\right) d z d S \\
& -\int_{S_{m}}(q \delta w) d S-\int_{c_{\sigma}} \int_{-h}^{h}\left[\bar{T}_{1} \delta u_{1}^{(k)}+\bar{T}_{2} \delta u_{2}^{(k)}+\bar{T}_{z} \delta u_{z}^{(k)}\right] d s d z=0
\end{aligned}
$$

where $\delta$ is the variational operator; all other symbols have been defined in Section 2.

Substituting (1) and (2) into (14) and integrating across the plate thickness yields the 2D statement of virtual work

$$
\begin{aligned}
& \int_{S_{m}}\left(N_{1} \delta u_{, 1}+\right. N_{2} \delta v, 2+N_{12}\left(\delta u_{, 2}+\delta v_{, 1}\right)+M_{1} \delta \theta_{1,1}+M_{2} \delta \theta_{2,2}+M_{12}\left(\delta \theta_{1,2}+\delta \theta_{2,1}\right) \\
&+Q_{1}\left(\delta w_{, 1}\right.\left.\left.+\delta \theta_{1}\right)+Q_{2}\left(\delta w_{, 2}+\delta \theta_{2}\right)-q \delta w\right) d S-\int_{C_{\sigma}}\left(\bar{N}_{1 n} \delta u+\bar{N}_{2 n} \delta v+\bar{Q}_{z n} \delta w+\bar{M}_{1 n} \delta \theta_{y}+\bar{M}_{2 n} \delta \theta_{x}\right) d s \\
&+\int_{S_{m}}\left(M_{1}^{\phi} \delta \psi_{1,1}+M_{2}^{\phi} \delta \psi_{2,2}+M_{12}^{\phi} \delta \psi_{1,2}+M_{21}^{\phi} \delta \psi_{2,1}+Q_{1}^{\phi} \delta \psi_{1}+Q_{2}^{\phi} \delta \psi_{2}\right) d S \\
&-\int_{C_{\sigma}}\left(\bar{M}_{1 n}^{\phi} \delta \psi_{1}+\bar{M}_{2 n}^{\phi} \delta \psi_{2}\right) d s=0, \quad(15)
\end{aligned}
$$

where the underlined terms correspond to the zigzag kinematics contributions. In Equation (15), the membrane stress resultants and conjugate strain measures are

$$
\boldsymbol{N}_{m}^{T} \equiv\left\{N_{1}, N_{2}, N_{12}\right\}=\int_{-h}^{h}\left\{\sigma_{11}^{(k)}, \sigma_{22}^{(k)}, \tau_{12}^{(k)}\right\} d z, \quad \boldsymbol{e}_{m}^{T} \equiv\left\{u_{, 1}, v_{, 2}, u_{, 2}+v_{, 1}\right\} .
$$

Likewise, the bending stress resultants and conjugate strain measures are

$$
\begin{aligned}
\boldsymbol{M}_{b}^{T} & \equiv\left\{M_{1}, M_{1}^{\phi}, M_{2}, M_{2}^{\phi}, M_{12}, M_{12}^{\phi}, M_{21}^{\phi}\right\} \\
& =\int_{-h}^{h}\left\{z \sigma_{11}^{(k)}, \phi_{1}^{(k)} \sigma_{11}^{(k)}, z \sigma_{22}^{(k)}, \phi_{2}^{(k)} \sigma_{22}^{(k)}, z \tau_{12}^{(k)}, \phi_{1}^{(k)} \tau_{12}^{(k)}, \phi_{2}^{(k)} \tau_{12}^{(k)}\right\} d z, \\
\boldsymbol{e}_{b}^{T} & \equiv\left\{\theta_{1,1}, \psi_{1,1}, \theta_{2,2}, \psi_{2,2}, \theta_{1,2}+\theta_{2,1}, \psi_{1,2}, \psi_{2,1}\right\},
\end{aligned}
$$

and the transverse shear stress resultants and conjugate strain measures are

$$
\boldsymbol{Q}_{s}^{T} \equiv\left\{Q_{2}, Q_{2}^{\phi}, Q_{1}, Q_{1}^{\phi}\right\}=\int_{-h}^{h}\left\{\tau_{2 z}^{(k)}, \beta_{2}^{(k)} \tau_{2 z}^{(k)}, \tau_{1 z}^{(k)}, \beta_{1}^{(k)} \tau_{1 z}^{(k)}\right\} d z, \boldsymbol{e}_{s}^{T} \equiv\left\{w_{, 2}+\theta_{2}, \psi_{2}, w_{, 1}+\theta_{1}, \psi_{1}\right\} .
$$

The force and moment resultants due to the prescribed tractions have the form

$$
\left\{\bar{N}_{1 n}, \bar{N}_{2 n}, \bar{Q}_{z n}, \bar{M}_{1 n}, \bar{M}_{2 n}, \bar{M}_{1 n}^{\phi}, \bar{M}_{2 n}^{\phi}\right\}=\int_{-h}^{h}\left\{\bar{T}_{1}, \bar{T}_{2}, \bar{T}_{z}, z \bar{T}_{1}, z \bar{T}_{2}, \phi_{1}^{(k)} \bar{T}_{1}, \phi_{2}^{(k)} \bar{T}_{2}\right\} d z .
$$

Integrating (15) by parts results in seven equilibrium equations and associated boundary conditions. 
The equilibrium equations are

$$
\begin{array}{rrrrl}
\delta u: & N_{1,1}+N_{12,2}=0, & \delta v: & N_{12,1}+N_{2,2}=0, & \delta w: Q_{1,1}+Q_{2,2}+q=0, \\
\delta \theta_{1}: & M_{1,1}+M_{12,2}-Q_{1}=0, & \delta \theta_{2}: & M_{12,1}+M_{2,2}-Q_{2}=0, \\
\delta \psi_{1}: & M_{1,1}^{\phi}+M_{12,2}^{\phi}-Q_{1}^{\phi}=0, & \delta \psi_{2}: & M_{21,1}^{\phi}+M_{2,2}^{\phi}-Q_{2}^{\phi}=0 .
\end{array}
$$

The kinematic and force boundary conditions are given by

$$
\begin{array}{rllcl}
u=\bar{u} & \text { on } C_{u} & \text { or } & N_{1} n_{1}+N_{12} n_{2}=\bar{N}_{1 n} & \text { on } C_{\sigma}, \\
v=\bar{v} & \text { on } C_{u} & \text { or } & N_{12} n_{1}+N_{2} n_{2}=\bar{N}_{2 n} & \text { on } C_{\sigma}, \\
w=\bar{w} & \text { on } C_{u} & \text { or } & Q_{1} n_{1}+Q_{2} n_{2}=\bar{Q}_{z n} & \text { on } C_{\sigma}, \\
\theta_{1}=\bar{\theta}_{1} & \text { on } C_{u} & \text { or } & M_{1} n_{1}+M_{12} n_{2}=\bar{M}_{1 n} & \text { on } C_{\sigma}, \\
\theta_{2}=\bar{\theta}_{2} & \text { on } C_{u} & \text { or } & M_{12} n_{1}+M_{2} n_{2}=\bar{M}_{2 n} & \text { on } C_{\sigma}, \\
\psi_{1}=\bar{\psi}_{1} & \text { on } C_{u} & \text { or } & M_{1}^{\phi} n_{1}+M_{12}^{\phi} n_{2}=\bar{M}_{1 n}^{\phi} & \text { on } C_{\sigma}, \\
\psi_{2}=\bar{\psi}_{2} & \text { on } C_{u} & \text { or } & M_{21}^{\phi} n_{1}+M_{2}^{\phi} n_{2}=\bar{M}_{2 n}^{\phi} & \text { on } C_{\sigma},
\end{array}
$$

where $n_{1}=\cos \left(x_{1}, n\right)$ and $n_{2}=\cos \left(x_{2}, n\right)$ are the components (direction cosines) of the unit outward normal vector to the cylindrical plate edges.

The plate constitutive relations are derived by using Equations (2) and (3) with (16)-(18), and integrating over the laminate thickness. The resulting constitutive relations of the new zigzag plate theory are expressed in matrix form as

$$
\left\{\begin{array}{c}
\boldsymbol{N}_{m} \\
\boldsymbol{M}_{b} \\
\boldsymbol{Q}_{s}
\end{array}\right\}=\left[\begin{array}{ccc}
\boldsymbol{A} & \boldsymbol{B} & \mathbf{0} \\
\boldsymbol{B}^{T} & \boldsymbol{D} & \mathbf{0} \\
\mathbf{0} & \mathbf{0} & \boldsymbol{G}
\end{array}\right]\left\{\begin{array}{l}
\boldsymbol{e}_{m} \\
\boldsymbol{e}_{b} \\
\boldsymbol{e}_{s}
\end{array}\right\}
$$

The expressions for the components of the stiffness matrices $\boldsymbol{A} \equiv\left[A_{i j}\right](i, j=1, \ldots, 3), \boldsymbol{B} \equiv\left[B_{i j}\right]$ $(i=1, \ldots, 3, j=1, \ldots, 7), \boldsymbol{D} \equiv\left[D_{i j}\right](i, j=1, \ldots, 7)$, and $\boldsymbol{G} \equiv\left[G_{i j}\right](i, j=1, \ldots, 4)$ are given in the Appendix.

Introducing (22) into (20) results in seven second-order partial differential equilibrium equations in terms of seven kinematic variables, giving rise to a $14^{\text {th }}$-order theory. The equilibrium equations can be solved exactly or approximately depending on the complexity of the material lay-up, boundary conditions, and loading. In addition, because the highest partial derivative appearing in the strain measures in (15) are first-order, computationally efficient $C^{0}$-continuous plate finite elements can be developed, thus enabling application of this refined zigzag theory in large-scale analyses of complex aerospace structures.

\section{Example problems and results}

To determine the accuracy of the present zigzag plate theory, analytic solutions for simply supported and cantilevered rectangular laminates are derived and detailed distributions of the displacements and stresses are examined. The rectangular laminates are defined on the domain $x_{1} \in[0, a], x_{2} \in[0, b], z \in[-h, h]$. 
Example 1. A simply supported rectangular plate is subjected to the sinusoidal transverse pressure $q\left(x_{1}, x_{2}\right)=q_{0} \sin \left(\pi x_{1} / a\right) \sin \left(\pi x_{2} / b\right)$. The simply supported boundary conditions are obtained from (21). For cross-ply and uniaxial laminates, the kinematic and force boundary conditions along $x_{1} \in[0, a]$ are

$$
v=w=\theta_{2}=\psi_{2}=0, \quad N_{1}=M_{1}=M_{1}^{\phi}=0,
$$

and along $x_{2} \in[0, b]$,

$$
u=w=\theta_{1}=\psi_{1}=0, \quad N_{2}=M_{2}=M_{2}^{\phi}=0 .
$$

For this set of boundary conditions, the exact solutions are obtained by the trigonometric expansions

$w=W \sin \frac{\pi x_{1}}{a} \sin \frac{\pi x_{2}}{b}, \quad\left\{\begin{array}{c}u \\ \theta_{1} \\ \psi_{1}\end{array}\right\}=\left\{\begin{array}{c}U \\ \Theta_{1} \\ \Psi_{1}\end{array}\right\} \cos \frac{\pi x_{1}}{a} \sin \frac{\pi x_{2}}{b}, \quad\left\{\begin{array}{c}v \\ \theta_{2} \\ \psi_{2}\end{array}\right\}=\left\{\begin{array}{c}V \\ \Theta_{2} \\ \Psi_{2}\end{array}\right\} \sin \frac{\pi x_{1}}{a} \cos \frac{\pi x_{2}}{b}$,

where $\left\{U, V, W, \Theta_{1}, \Theta_{2}, \Psi_{1}, \Psi_{2}\right\}$ are the unknown amplitudes of the kinematic variables that are determined from satisfaction of the equilibrium equations.

For antisymmetric angle-ply laminates, the kinematic and force boundary conditions along $x_{1} \in[0, a]$ are

$$
u=w=\theta_{2}=\psi_{2}=0, \quad N_{12}=M_{1}=M_{1}^{\phi}=0,
$$

and along $x_{2} \in[0, b]$

$$
v=w=\theta_{1}=\psi_{1}=0, \quad N_{12}=M_{2}=M_{2}^{\phi}=0 .
$$

Thus, the trigonometric expansions that satisfy (25a) and (25b) exactly differ from those in (24) only for the $u$ and $v$ variables, and they are given by

$$
u=U \sin \frac{\pi x_{1}}{a} \cos \frac{\pi x_{2}}{b}, \quad v=V \cos \frac{\pi x_{1}}{a} \sin \frac{\pi x_{2}}{b} .
$$

Example 2. A cantilevered rectangular plate is clamped along a single edge $\left(x_{1}=0\right)$, free along the other edges, and subjected to the uniform transverse pressure $q\left(x_{1}, x_{2}\right)=q_{0}$. The clamped boundary conditions along $x_{1}=0$ are

$$
u=v=w=\theta_{1}=\theta_{2}=\psi_{1}=\psi_{2}=0 .
$$

Unlike the previous zigzag theories where the clamped boundary conditions result in erroneous solutions for transverse shear stresses and forces that vanish along the clamped edges, the solutions of the present theory do not possess such anomalies. For instance, along the clamped boundary at $x_{1}=0$, the kinematic constraints in (27a), with the use of (2e), (13b), and (18), give rise to the following transverse shear stresses, $\tau_{1 z}^{(k)}\left(0, x_{2}, z\right)$, and force, $Q_{1}\left(0, x_{2}\right)$ :

$$
\left\{\begin{array}{c}
\tau_{1 z}^{(k)} \\
Q_{1}
\end{array}\right\}_{\left(x_{1}=0\right)}=\left\{\begin{array}{c}
Q_{11}^{(k)} \\
\int_{-h}^{h} Q_{11}^{(k)} d z
\end{array}\right\} w_{, 1}\left(0, x_{2}\right)
$$

where, in general, $w, 1\left(0, x_{2}\right) \neq 0$.

The traction-free boundary conditions along the edge $x_{1}=a$ are

$$
N_{1}=N_{12}=M_{1}=M_{12}=Q_{1}=M_{1}^{\phi}=M_{12}^{\phi}=0,
$$




\begin{tabular}{|clccc|}
\hline & Lamina material & $E_{1}^{(k)}, E_{2}^{(k)}, E_{3}^{(k)}$ & $v_{12}^{(k)}, v_{13}^{(k)}, v_{23}^{(k)}$ & $G_{12}^{(k)}, G_{13}^{(k)}, G_{23}^{(k)}$ \\
\hline \multirow{2}{*}{$\mathrm{C}$} & Carbon-epoxy & $1.579 \times 10^{2}$ & 0.32 & 5.930 \\
& unidirectional composite & 9.584 & 0.32 & 5.930 \\
& & 9.584 & 0.49 & 3.227 \\
\multirow{2}{*}{$\mathrm{H}$} & Titanium honeycomb core & $1.915 \times 10^{-1}$ & $0.658 \times 10^{-2}$ & $4.227 \times 10^{-8}$ \\
& & $1.915 \times 10^{-1}$ & $0.643 \times 10^{-6}$ & $3.651 \times 10^{-1}$ \\
& & $0.643 \times 10^{-6}$ & 1.248 \\
\hline $\mathrm{P}$ & PVC core & $E^{(k)}=1.040 \times 10^{-1}$ & $v^{(k)}=0.3$ & \\
\hline $\mathrm{T}$ & Titanium & $E^{(k)}=1.041 \times 10^{2}$ & $v^{(k)}=0.31$ & \\
\hline
\end{tabular}

Table 1. Mechanical properties of orthotropic materials $(\mathrm{C}$ and $\mathrm{H})$ and isotropic material (P and T). The Young's modulus $E$ and shear modulus $G$ are expressed in GPa.

\begin{tabular}{|llccc|}
\hline Laminate & $\begin{array}{c}\text { Normalized lamina } \\
\text { thickness, } h^{(k)} / h\end{array}$ & Lamina materials & Lamina orientation [ ${ }^{\circ}$ ] \\
$A$ & Cross-ply composite & $(0.5 / 0.5)$ & $(\mathrm{C} / \mathrm{C})$ & $(0 / 90)$ \\
$B$ & Uniaxial sandwich & $(0.1 / 0.8 / 0.1)$ & $(\mathrm{C} / \mathrm{P} / \mathrm{C})$ & $(0 / 0 / 0)$ \\
$F$ & Uniaxial sandwich & $(0.1 / 0.8 / 0.1)$ & $(\mathrm{T} / \mathrm{H} / \mathrm{T})$ & $(0 / 0 / 0)$ \\
$G$ & Angle-ply sandwich & $(0.05 / 0.05 / 0.8 / 0.05 / 0.05)$ & $(\mathrm{C} / \mathrm{C} / \mathrm{P} / \mathrm{C} / \mathrm{C})$ & $(30 /-45 / 0 / 45 /-30)$ \\
\hline
\end{tabular}

Table 2. Laminate stacking sequences (lamina sequence is in the positive $z$ direction).

and along the edge $x_{1} \in[0, b]$

$$
N_{12}=N_{2}=M_{12}=M_{2}=Q_{2}=M_{12}^{\phi}=M_{2}^{\phi}=0 .
$$

For both example problems, various laminates are considered with the emphasis on relatively thick laminated composite and sandwich plates having span-to-thickness ratio $a / 2 h=b / 2 h=5$. The mechanical material properties are listed in Table 1, and Table 2 summarizes the stacking sequences of the laminates.

The example problems include: a two-layer, cross-ply carbon-epoxy laminate, labeled laminate $A$; a three-layer sandwich laminate, laminate $B$, having uniaxial carbon-epoxy face sheets and a thick, closedcell polyvinyl chloride (PVC) core, where PVC is represented as an isotropic material; a sandwich laminate with two-layered titanium face sheets and a thick titanium honeycomb core, laminate $F$; and a five-layer, angle-ply sandwich laminate with carbon-epoxy face sheets and a thick PVC core, laminate $G$. Additional sandwich laminates that involve very thin face sheets have been studied in [Tessler et al. 2009].

For comparison purposes, several analytic and finite element solutions were also obtained for the corresponding boundary-value problems using 3D elasticity theory [Pagano 1969; Noor and Burton 1990], FSDT, the theory of Di Sciuva [1984a], and MSC/MD-NASTRAN finite element code [MSC 2006]. Note that application of FSDT generally requires the use of shear correction factors. For laminated composites, 
lamination-dependent shear correction factors have been shown to provide relatively accurate deflection predictions (for examples, refer to [Vlachoutsis 1992; Reddy and Vijayakumar 1995]). Yet, such shear corrections fail to furnish substantial improvements for the normal strain and stress predictions. Presently, to establish a common framework reference for FSDT, a shear correction factor $k^{2}=\frac{5}{6}$, appropriate for homogeneous plates, was used throughout.

For the simply supported cross-ply and uniaxial laminates, the exact solutions for the FSDT and Di Sciuva zigzag theory were obtained using the trigonometric functions in (24) (excluding those functions for $\psi_{\alpha}(\alpha=1,2)$ which do not appear in FSDT). For the angle-ply antisymmetric laminates, Di Sciuva theory permits only approximate solutions to be determined. Presently, the Rayleigh-Ritz method was employed, where the kinematic variables were approximated with suitable Gram-Schmidt polynomials [Arfken 1985] that satisfy the kinematic boundary conditions, (26), exactly (refer to [Tessler et al. 2009] for the particular expressions of the Gram-Schmidt polynomials used). Furthermore, for the simply supported cross-ply and angle-ply antisymmetric laminates, exact 3D elasticity solutions were obtained using the solution procedures developed by Pagano [1969] and Noor and Burton [1990].

For the cantilevered laminates, approximate solutions corresponding to Di Sciuva and refined (present) zigzag theories were developed using the Rayleigh-Ritz method. Here the displacement approximations are based on the Gram-Schmidt polynomials, using seven functions along the $x_{1}$ axis and five along the $x_{2}$ axis (for details, refer to [Tessler et al. 2009]). Furthermore, for the cantilevered plate (laminate $B$ ), a high-fidelity 3D finite element solution was obtained by using MSC/MD-NASTRAN. The model is regularly discretized and is comprised of three elements through the thickness for each face sheet, six elements through the core thickness, and sixty subdivisions along each span direction, with a total of 43,200 HEXA20, linear-strain elements.

The numerical and graphical results that follow are labeled as:

- 3D Elasticity (3D elasticity solutions using procedures developed by Pagano [1969] for cross-ply laminates and by Noor and Burton [1990] for angle-ply antisymmetric laminates).

- FSDT (first-order shear deformation theory, $k^{2}=\frac{5}{6}$ ).

- Zigzag (D) [Di Sciuva 1984a].

- Zigzag (R) (present, refined zigzag theory).

- 3D FEM (3D FEM solution using MSC/MD-NASTRAN [MSC 2006]).

- Zigzag (R-E) (transverse shear stresses obtained by way of integrating 3D elasticity equilibrium equations, using the normal and in-plane shear stresses derived from the refined zigzag theory).

Comparisons of the maximum deflection and maximum top-surface displacement are presented for the simply supported square laminates $(a / 1 h=b / 2 h=5)$ in Tables 3 and 4 . These results demonstrate that both zigzag theories predict accurate plate displacements as compared to the $3 \mathrm{D}$ elasticity solution, with the refined theory achieving slightly more accurate predictions. The laminate $G$ case is somewhat pathological for Zigzag (D), because the solution in this case is only approximate and has not converged. The deflections predicted by FSDT are generally overly stiff; however, they are expected to improve substantially with the use of lamination-dependent shear correction factors. In Table 5, the range of applicability of the various theories is examined by comparing the maximum (central) plate deflection for a simply supported sandwich laminate $B$, where the solutions correspond to a span-to-thickness 


\begin{tabular}{|ccrrrr|}
\hline Laminate & $\begin{array}{c}\text { Normalization factor } \\
\left(10^{2} D_{11} q_{0} a^{4}\right)\end{array}$ & 3D elasticity & FSDT & Zigzag (D) & Zigzag (R) \\
& $8.147 \times 10^{-2}$ & 1.228 & 1.278 & 1.170 & 1.219 \\
$B$ & $7.502 \times 10^{-2}$ & 29.761 & 2.731 & 29.769 & 29.785 \\
$F$ & $5.444 \times 10^{-2}$ & 1.331 & 0.389 & 1.332 & 1.333 \\
$G$ & $3.551 \times 10^{-2}$ & 14.124 & 1.055 & 12.734 & 14.105 \\
\hline
\end{tabular}

Table 3. Normalized maximum (central) deflection, $\bar{w}=\left(10^{2} D_{11} / q_{0} a^{4}\right) w(a / 2, b / 2)$, for simply supported laminates subjected to sinusoidal transverse pressure loading.

\begin{tabular}{|cccccc|}
\hline Laminate & $\begin{array}{c}\text { Normalization factor } \\
\left(10^{3} D_{11} / q_{o} a^{4}\right)\end{array}$ & 3D Elasticity & FSDT & Zigzag (D) & Zigzag (R) \\
$A$ & $8.147 \times 10^{-1}$ & 4.233 & 4.152 & 3.855 & 4.251 \\
$B$ & $7.502 \times 10^{-1}$ & 9.977 & 2.156 & 9.945 & 9.897 \\
$F$ & $5.444 \times 10^{-1}$ & 0.643 & 0.796 & 0.646 & 0.649 \\
$G$ & $3.551 \times 10^{-1}$ & 3.908 & 0.704 & 1.295 & 3.845 \\
\hline
\end{tabular}

Table 4. Normalized maximum (top surface) displacement along the $x_{1}$ axis $\bar{u}_{1}=\left(10^{3} D_{11} / q_{o} a^{4}\right) \times u_{1}^{(N)}(a, b / 2, h)$ of simply supported laminates subjected to sinusoidal transverse pressure loading.

\begin{tabular}{|cccccc|}
\hline $\begin{array}{c}\text { Span-to-thickness } \\
\text { ratio }\end{array}$ & $\begin{array}{c}\text { Normalization factor } \\
\left(10^{2} D_{11} / q_{0} a^{4}\right)\end{array}$ & 3D Elasticity & FSDT & Zigzag (D) & Zigzag (R) \\
4 & $1.832 \times 10^{-1}$ & 42.420 & 3.739 & 42.124 & 42.189 \\
10 & $4.668 \times 10^{-3}$ & 9.734 & 1.321 & 9.738 & 9.739 \\
20 & $2.931 \times 10^{-4}$ & 3.487 & 0.948 & 3.489 & 3.490 \\
50 & $7.502 \times 10^{-6}$ & 1.305 & 0.841 & 1.305 & 1.305 \\
100 & $4.688 \times 10^{-7}$ & 0.945 & 0.826 & 0.945 & 0.945 \\
200 & $2.931 \times 10^{-8}$ & 0.852 & 0.822 & 0.852 & 0.852 \\
\hline
\end{tabular}

Table 5. Normalized maximum (central) deflection, $\bar{w}=\left(10^{2} D_{11} / q_{0} a^{4}\right) w(a / 2, b / 2)$, for simply supported laminate $B$ subjected to sinusoidal transverse pressure loading and corresponding to various span-to-thickness ratios.

ratio in the range 4-200. These results are also plotted in Figure 3. It is observed that both zigzag theories predict accurate deflections for all span-to-thickness ratios examined, whereas FSDT is overly stiff, especially in the thick regime, as expected. Even for a relatively thin laminate $B(a / 2 h=50)$, FSDT underestimates the maximum deflection by about $36 \%$, which means that a much more significant shear correction factor would be required for FSDT for this type of laminate.

For the simply supported laminates, normalized through-the-thickness distributions for the in-plane displacement, $\bar{u}_{1}=\left(10^{4} D_{11} / q_{0} a^{4}\right) u_{1}^{(k)}(0, a / 2, z)$, the normal stress, $\bar{\sigma}_{11} \equiv\left(4 h^{2} / q_{0} a^{2}\right) \times \sigma_{11}^{(k)}(a / 2, a / 2, z)$, and the transverse shear stress, $\bar{\tau}_{1 z} \equiv\left(2 h / q_{0} a\right) \tau_{1 z}^{(k)}(0, a / 2, z)$, are depicted in Figures $4-11$. 

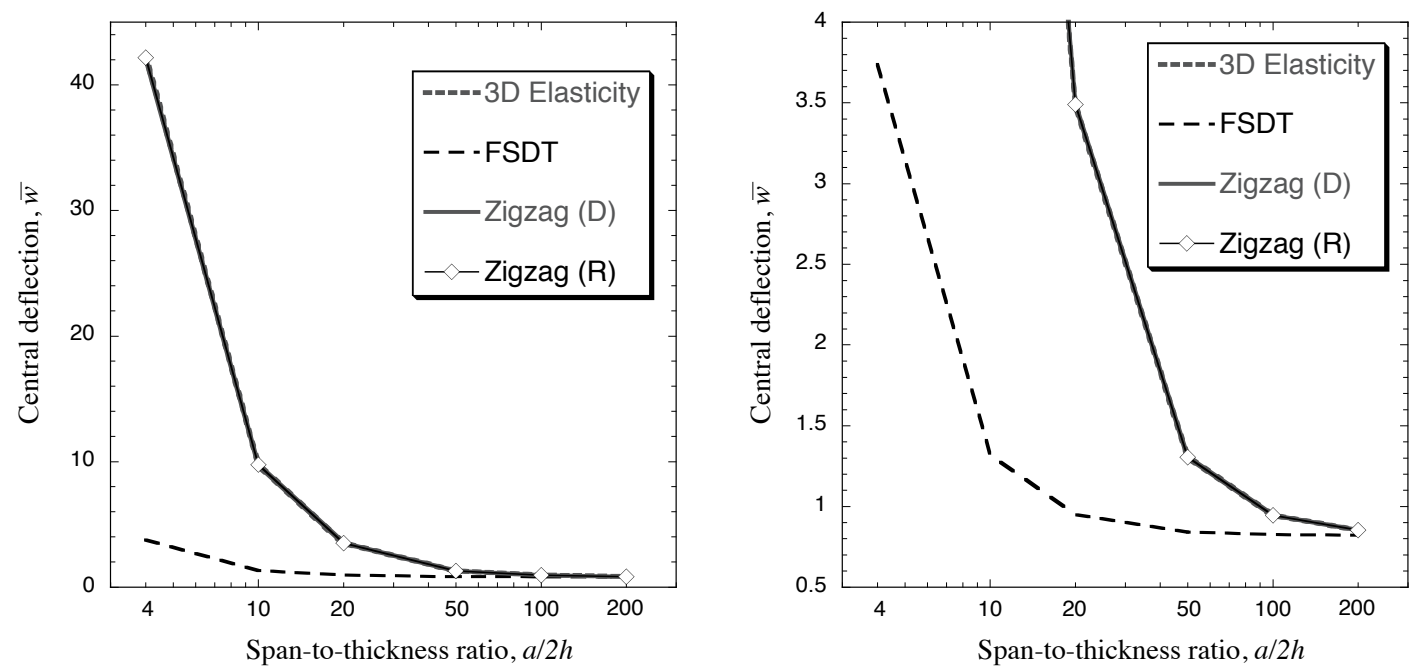

Figure 3. Normalized central deflection versus span-to-thickness ratio for simply supported laminate $B$ subjected to sinusoidal transverse pressure (left); zoomed view (right).

For laminate $A$ - an asymmetric cross-ply carbon-epoxy composite - the values of $\bar{u}_{1}$ and $\bar{\sigma}_{11}$ are accurately modeled by FSDT and the two zigzag theories (Figures 4 and 5), with Zigzag (D) producing slightly underestimated displacement near the top surface. The major differences in results for this laminate correspond to the $\bar{\tau}_{1 z}$ distribution (Figure 5), where both FSDT and Zigzag (R) produce piecewise constant stresses, whereas Zigzag (D) gives a uniform distribution for $\bar{\tau}_{1 z}$ significantly less accurate than the predictions of the other two theories. In addition, Zigzag $(\mathrm{R})$ theory provides a more accurate evaluation of the average transverse shear stress within each lamina than does FSDT. Also, the $\bar{\tau}_{1 z}$ stress, obtained by an equilibrium-based method, Zigzag (R-E), is best correlated with the 3D elasticity solution.
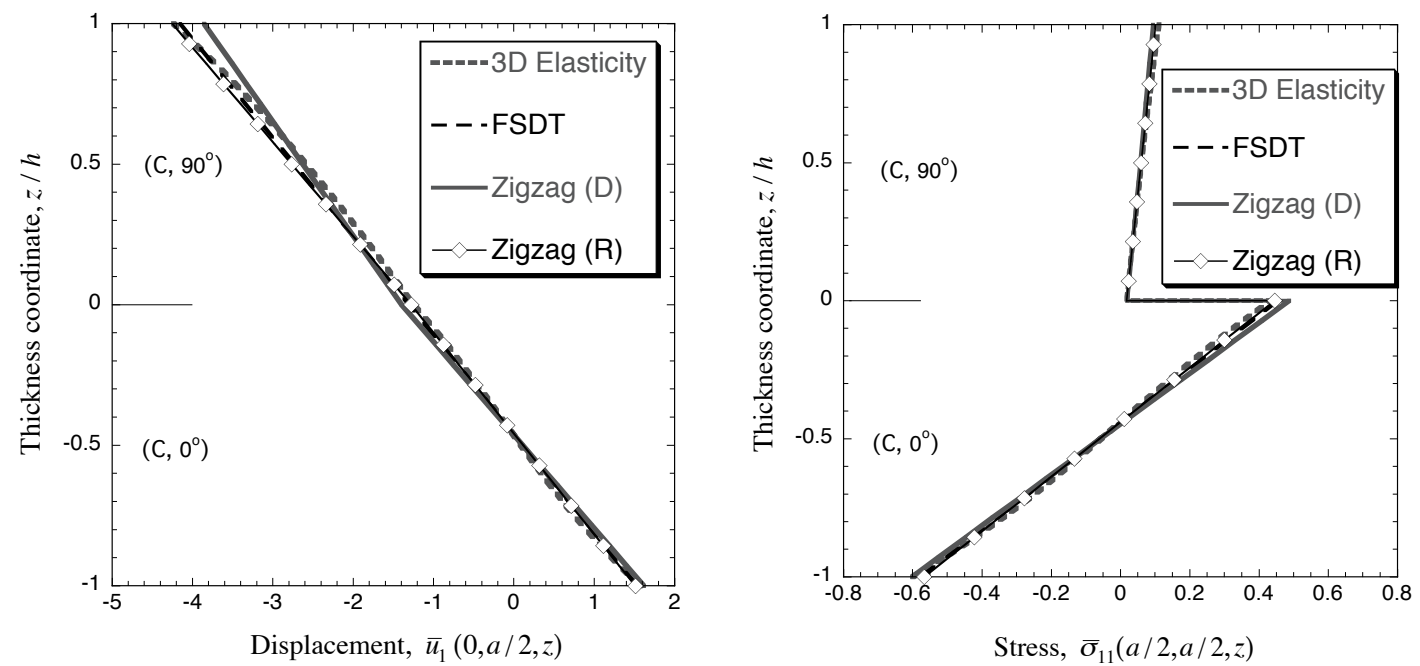

Figure 4. Normalized in-plane displacement (left) and normal stress (right) for simply supported laminate $A$ subjected to sinusoidal transverse pressure. 


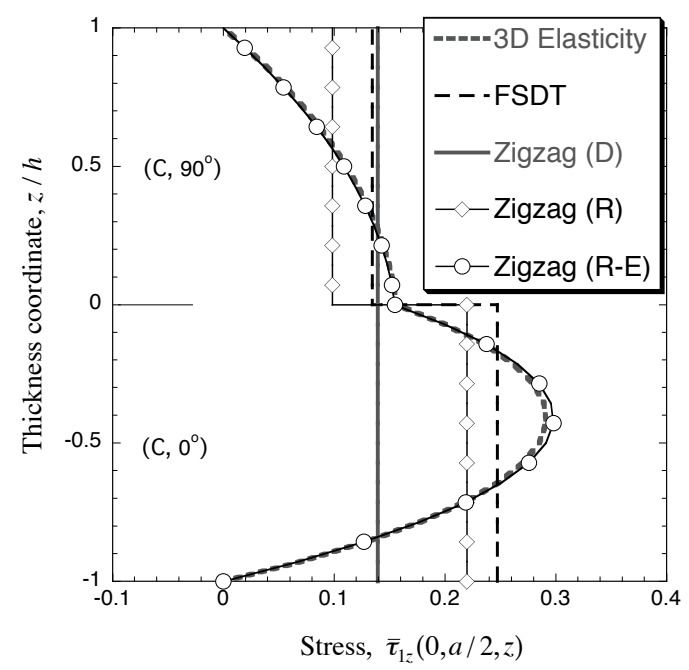

Figure 5. Normalized transverse shear stress for simply supported laminate $A$ subjected to sinusoidal transverse pressure.

The $\bar{u}_{1}, \bar{\sigma}_{11}$, and $\bar{\tau}_{1 z}$ results for sandwich panel $B$, which has constant-thickness carbon-epoxy face sheets and a PVC core, are provided in Figures 6 and 7. For laminate $B$, the top and bottom-surface values of the $\bar{u}_{1}$ displacement are significantly underestimated by FSDT which is only capable of a linear (average) distribution. Also, as shown in Figure 7, left, FSDT grossly underestimates the normal stress $\bar{\sigma}_{11}$ on the bounding surfaces where the greatest compression and tension occur. By contrast, Zigzag (R) yields accurate solutions of all response quantities examined. When the transverse shear stresses are evaluated from the constitutive relations, the theory provides the correct average values in the face sheets and in the core. Moreover, the equilibrium-based method, Zigzag (R-E), furnishes superior transverse shear stresses that are virtually indistinguishable from those of 3D elasticity. An

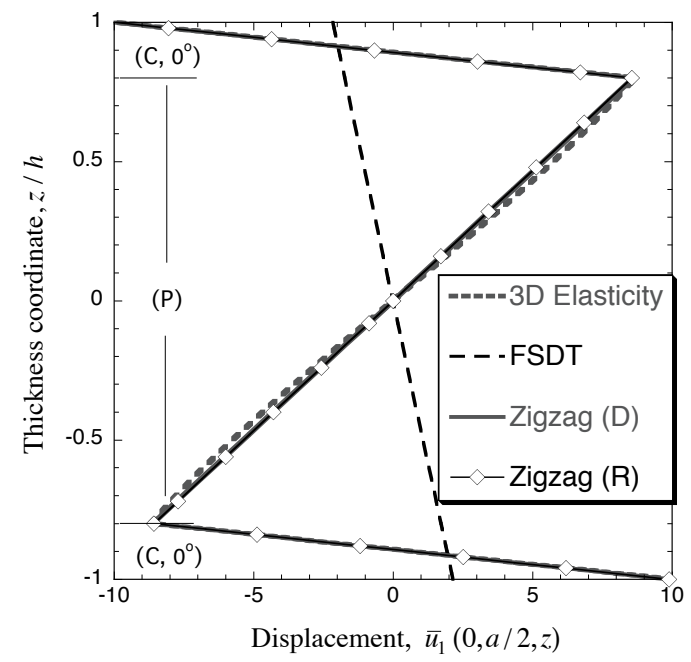

Figure 6. Normalized in-plane displacement for simply supported laminate $B$ subjected to sinusoidal transverse pressure. 

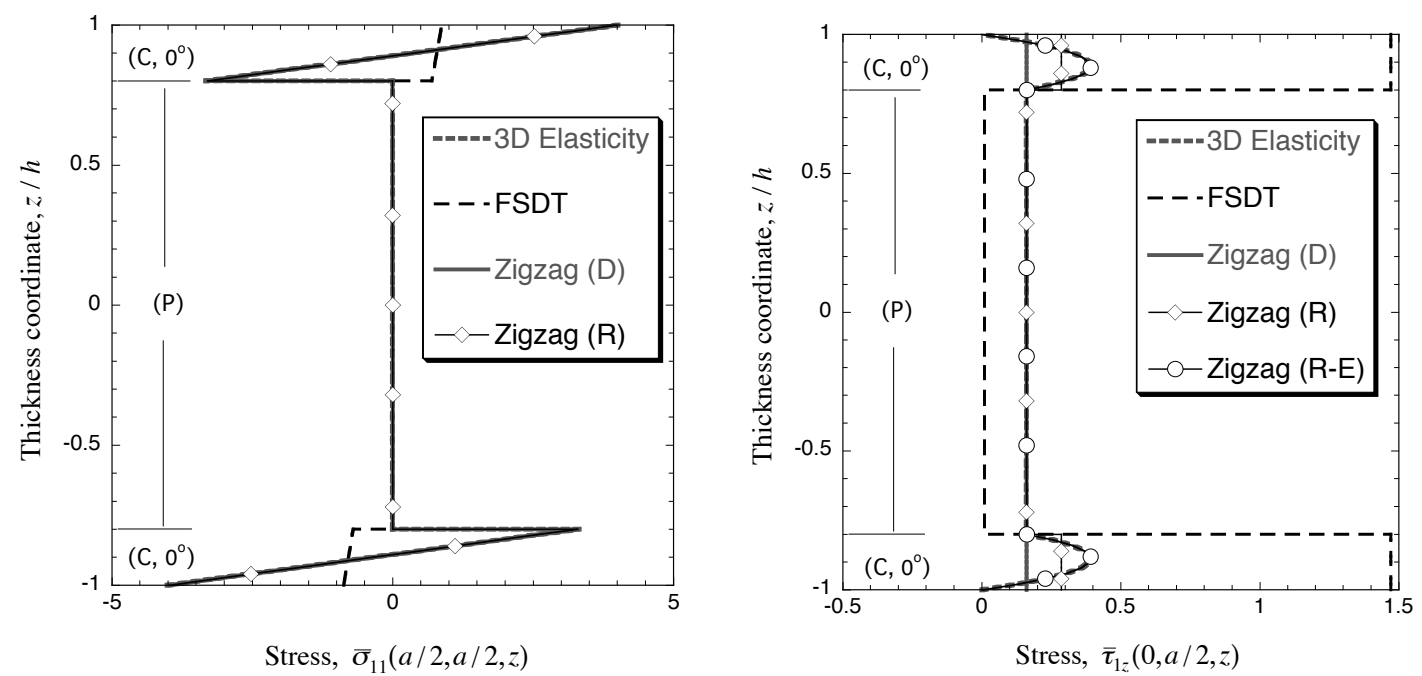

Figure 7. Normalized normal stress (left) and transverse shear stress (right) for simply supported laminate $B$ subjected to sinusoidal transverse pressure.

additional study evaluating the effect of face-sheet thinness in sandwich laminates on the distribution of displacements and stresses through the thickness can be found in [Tessler et al. 2009].

The displacement and stress results for laminate $F-$ a cross-ply sandwich with a lower degree of anisotropy than laminate $B$ - are depicted in Figures 8 and 9 . For this laminate, the zigzag effect of the in-plane displacement is somewhat less pronounced than for laminate $B$. As in the previous example, Zigzag $(\mathrm{R})$ yields highly accurate predictions of all response quantities. It is seen that FSDT provides overestimated values for the in-plane displacement on the bounding surfaces. Also, it is evident from Figure 9, left, that the integral of the shear stress over the thickness computed using the Zigzag (D) transverse shear stress would result in a significantly greater value than the exact shear force.
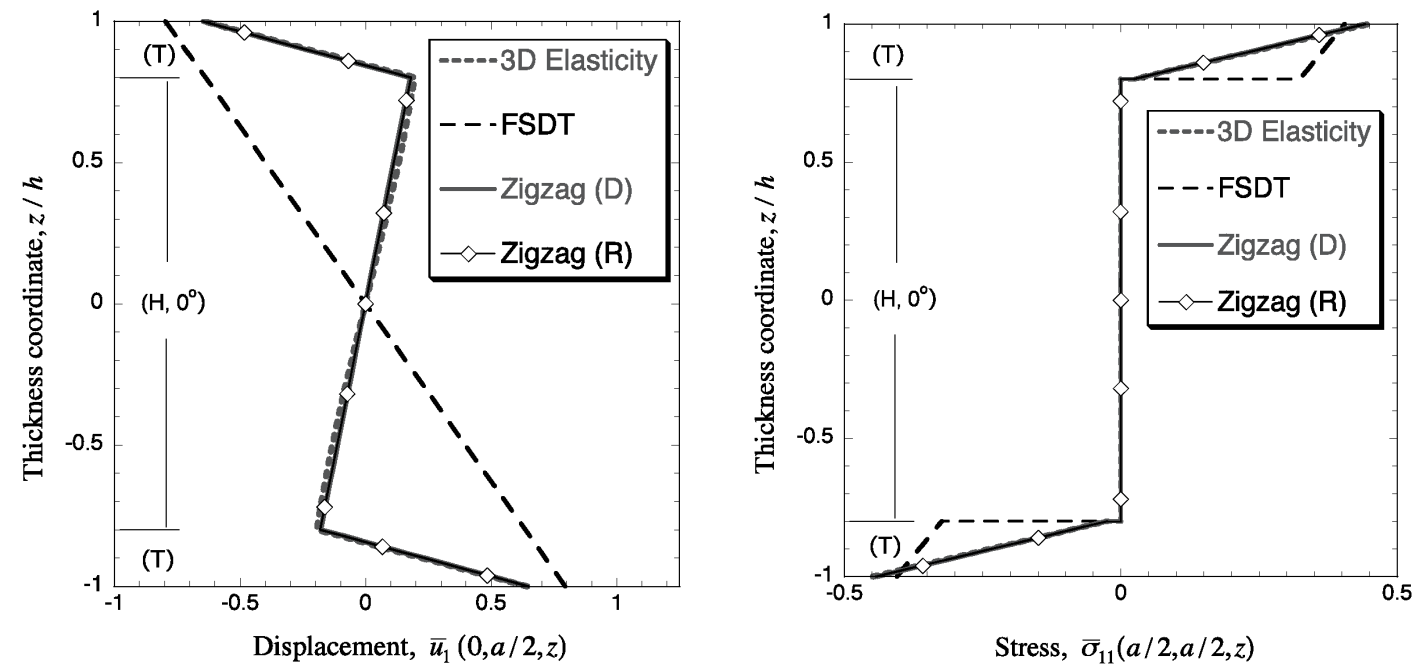

Figure 8. Normalized in-plane displacement (left) and normal stress (right) for simply supported laminate $F$ subjected to sinusoidal transverse pressure. 


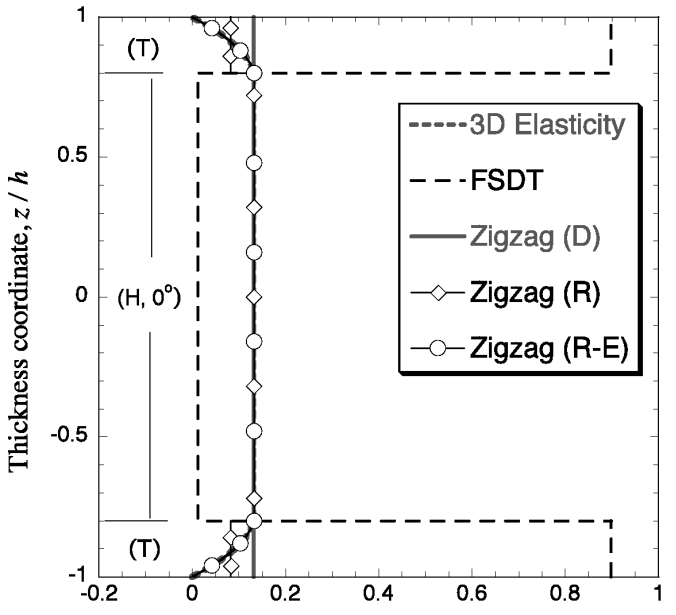

(a)

Stress, $\bar{\tau}_{1 z}(0, a / 2, z)$

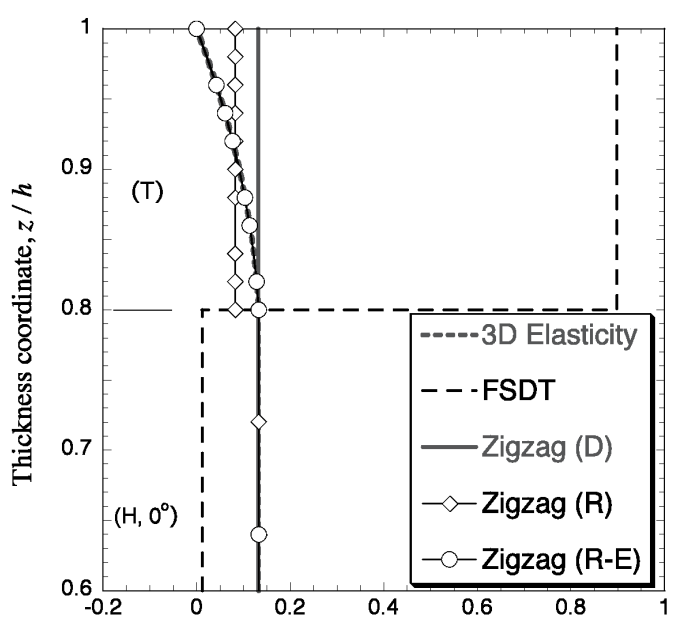

(b)

Stress, $\bar{\tau}_{1 z}(0, a / 2, z)$

Figure 9. Left: Normalized transverse shear stress for simply supported laminate $F$ subjected to sinusoidal transverse pressure. Right: detail near the top layer.

Figures 10 and 11 demonstrate the results for laminate $G$ - an angle-ply antisymmetric sandwich plate with multilayered face sheets. This is a highly challenging test case for any lamination theory. For this lamination, only an approximate solution can be obtained for Zigzag (D), requiring a large number of suitable shape functions to achieve a converged solution. Using the Rayleigh-Ritz method with the GramSchmidt polynomials approximating the kinematic variables (see the details in [Tessler et al. 2009]), a relatively inaccurate solution was obtained. Consequently, the Zigzag (D) results for the in-plane displacement and normal stress (Figure 10) are somewhat erroneous. On the other hand, Zigzag (R) enables an exact solution to be obtained for this problem, once again yielding highly accurate predictions of the
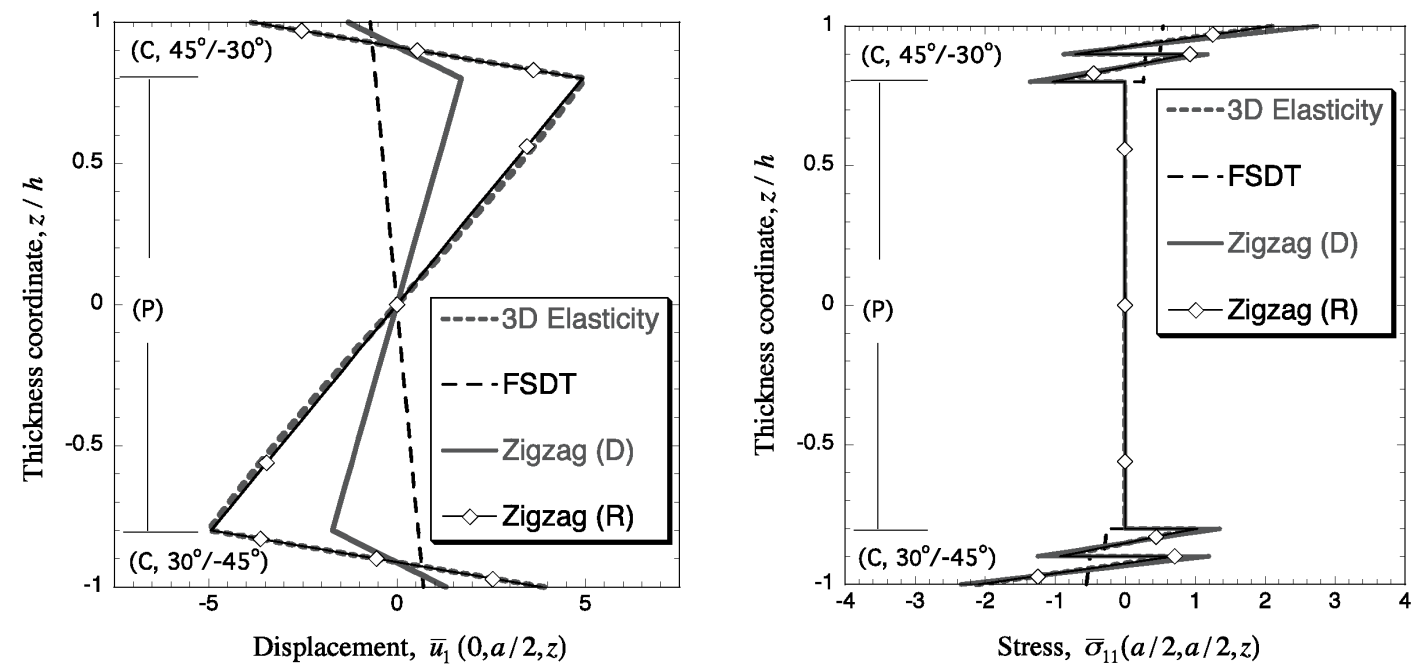

Figure 10. Normalized in-plane displacement (left) and normal stress (right) for simply supported laminate $G$ subjected to sinusoidal transverse pressure. 

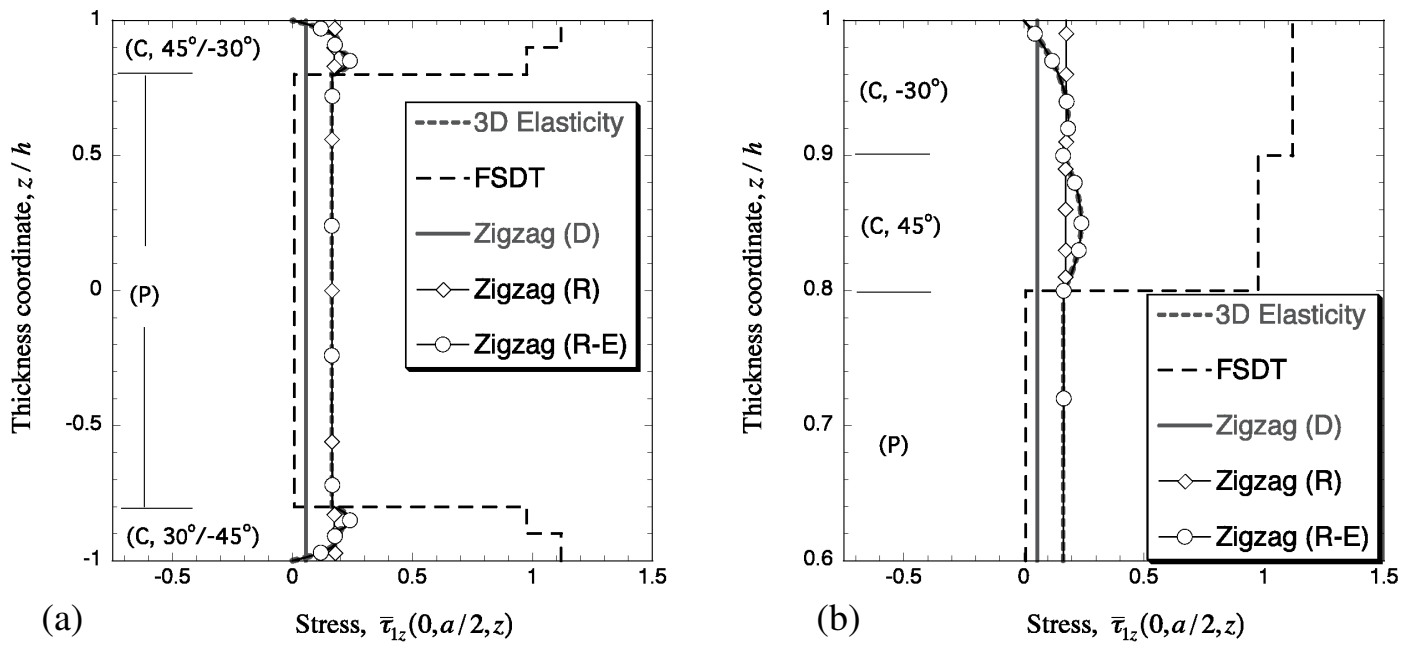

Figure 11. (a) Normalized transverse shear stress for simply supported laminate $G$ subjected to sinusoidal transverse pressure; (b) near the top layer.

response quantities. As in the previous examples, FSDT models the in-plane displacement response only in an average sense (a linear distribution through the thickness), leading to a significant underestimation (a nonconservative prediction) of the normal stress in the face sheets. Figure 11 demonstrates the highly accurate transverse shear stress obtained by the present theory, Zigzag (R), as compared to the other plate-theory solutions.

To examine the effect of clamped boundary conditions, a square cantilevered laminate $B$ under a uniform transverse pressure was examined. Table 6 summarizes the maximum deflection calculated using the three different theories and a 3D finite element solution that serves as a reference. The FSDT deflection is underestimated by an order of magnitude. The two zigzag theories give accurate results, with Zigzag (R) producing a somewhat superior deflection prediction.

For the cantilevered sandwich laminate $B$, normalized through-the-thickness distributions of the inplane displacement, $\bar{u}_{1}=\left(10^{4} D_{11} / q_{o} a^{4}\right) u_{1}^{(k)}(a, a / 2, z)$, the normal stress,

$$
\bar{\sigma}_{11}=\left((2 h)^{2} / q_{0} a^{2}\right) \times \sigma_{11}^{(k)}(a / 5, a / 2, z),
$$

and the transverse shear stress, $\bar{\tau}_{1 z}=\left(2 h / q_{0} a\right) \times \tau_{1 z}^{(k)}(a / 5, a / 2, z)$, are provided in Figures 12 and 13 . The stresses were computed near the clamped edge $\left(x_{1}=a / 5, x_{2}=a / 2\right)$ to allow for proper comparisons with the accurate stresses obtained from the 3D FEM analysis. For this problem, both zigzag theories produce accurate results; however, application of Zigzag $(\mathrm{R})$ resulted in superior predictions of transverse

\begin{tabular}{|ccccc|}
\hline Normalization factor $\left(10^{2} D_{11} / q_{0} a^{4}\right)$ & 3D FEM & FSDT & Zigzag (D) & Zigzag (R) \\
$7.502 \times 10^{-2}$ & 246.778 & 25.351 & 244.077 & 245.615 \\
\hline
\end{tabular}

Table 6. Normalized maximum (free-edge) deflection, $\bar{w}=\left(10^{2} D_{11} / q_{0} a^{4}\right) w(a / 2, b / 2)$, for cantilevered laminate $B$ subjected to uniform transverse pressure loading. 

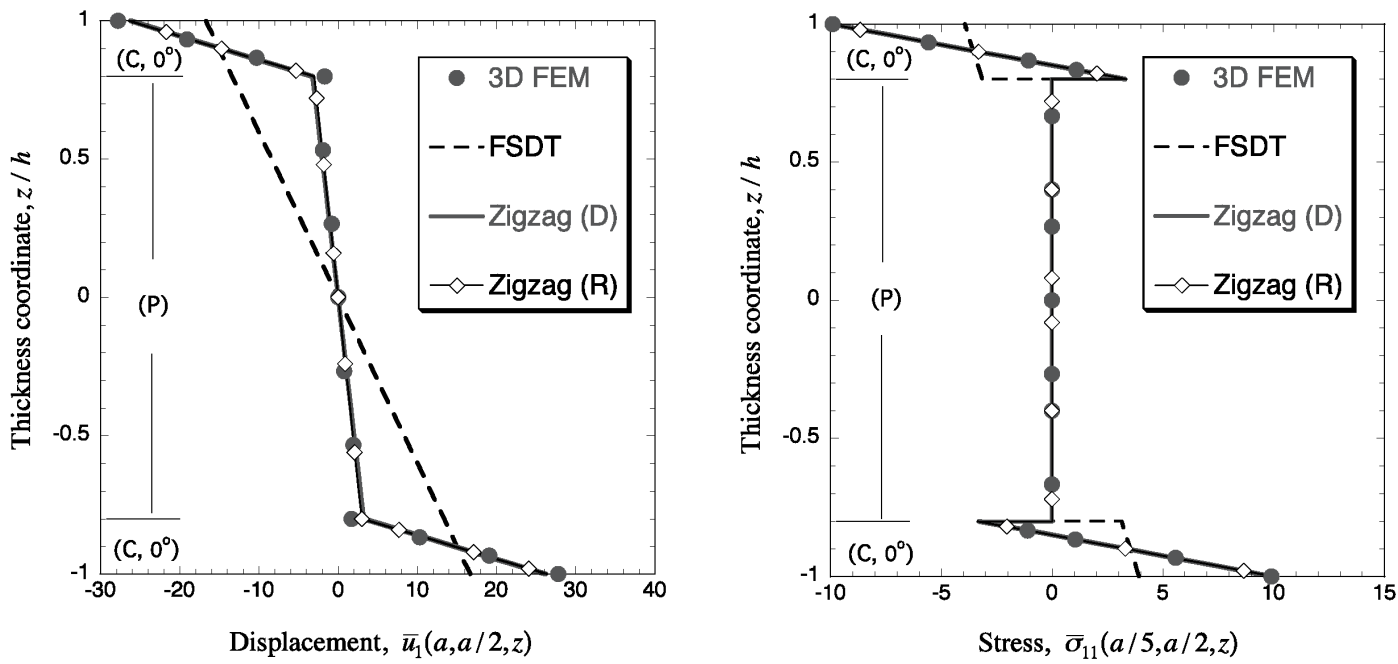

Figure 12. Normalized in-plane displacement (left) and normal stress (right) for cantilevered laminate $B$ subjected to uniform transverse pressure.

shear stresses. Finally, the normalized transverse shear force,

$$
\bar{Q}_{1}=\frac{1}{q_{0} a} \int_{-h}^{h} \tau_{1 z}^{(k)} d z,
$$

evaluated at $x_{2}=a / 2$, is plotted versus the normalized axial coordinate, $x_{1} / a$, as shown in Figure 14 . For this problem, both FSDT and Zigzag (R) predict the correct linear distribution, yielding a maximum value at the clamped edge and vanishing at the free edge. This contrasts with an erroneous Zigzag (D) solution that varies in a nonlinear manner across the span and which vanishes at the clamped edge.
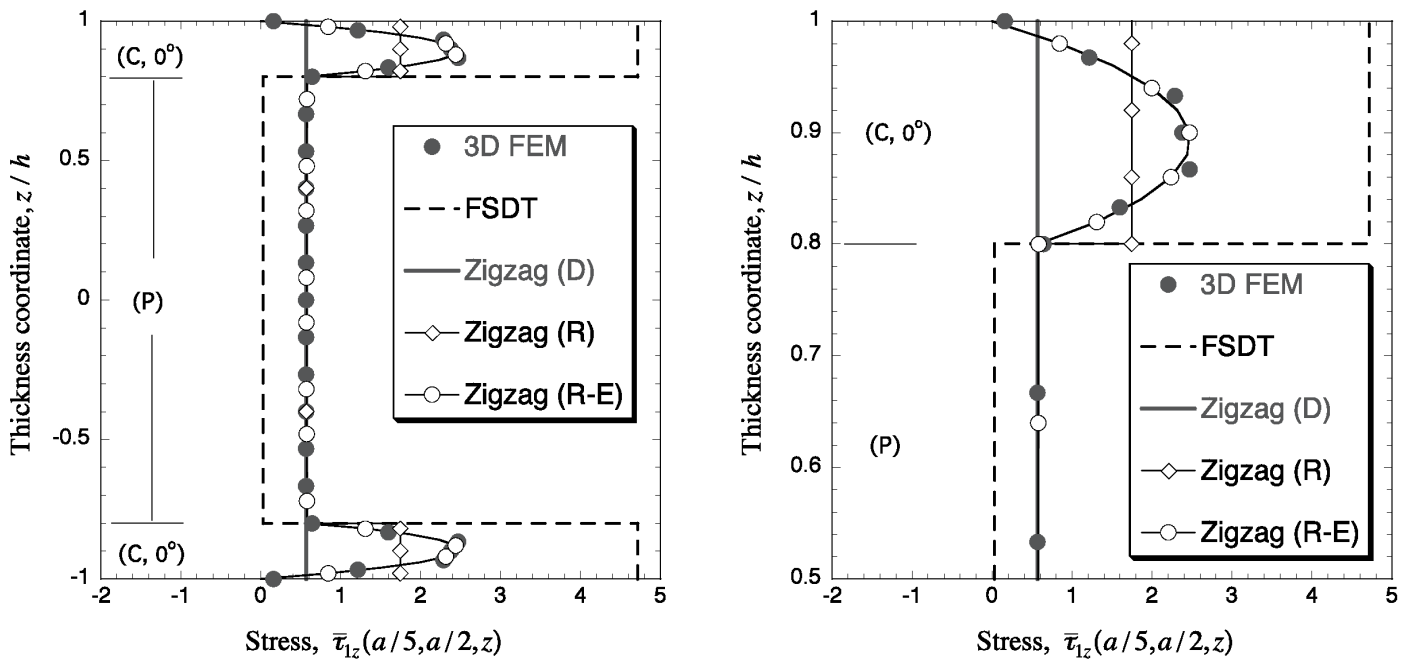

Figure 13. Normalized transverse shear stress for cantilevered laminate $B$ subjected to uniform transverse pressure. 


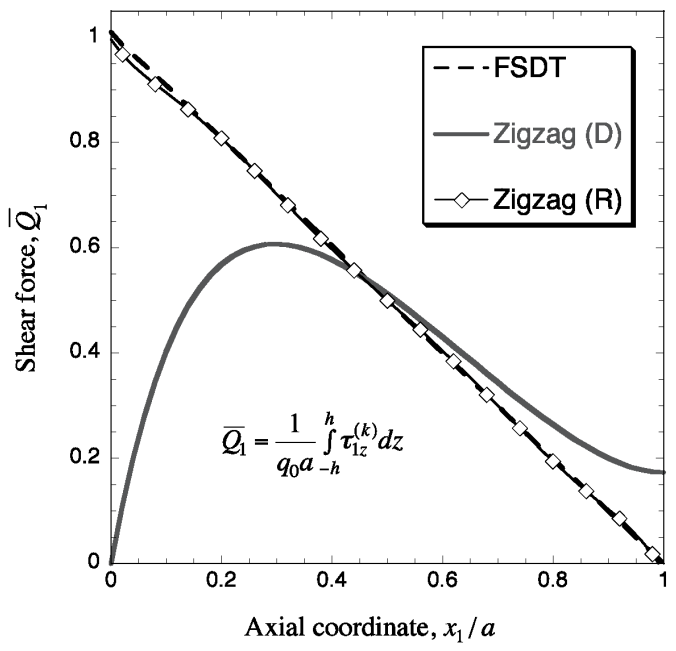

Figure 14. Normalized transverse shear force along centerline of cantilevered laminate $B$ subjected to uniform transverse pressure.

\section{Conclusions}

A variationally consistent and robust refined zigzag plate theory has been discussed and its predictive capability examined on laminated-composite and sandwich plates that exhibit a high degree of transverse shear flexibility, anisotropy, and heterogeneity. In this refined theory, a first-order shear deformation theory is used as a baseline for the kinematic assumptions with a set of novel piecewise-continuous zigzag displacements added to the in-plane displacement components. The resulting kinematic field is independent of the number of material layers, and the zigzag displacements are defined by requiring only partial lamina-interface continuity requirements of transverse shear stresses. The force equilibrium equations, boundary conditions, and strain-displacement relations are completely consistent with respect to the virtual work principle, and transverse shear correction factors are not required. The refined zigzag theory is better suited for engineering practice than previous similar theories because of its relative simplicity and its ability to model accurately the transverse shear and in-plane deformations of the individual laminae in a physically realistic manner. Unlike other similar theories, meaningful in-plane and transverse shear stresses are obtained directly from the constitutive equations, in a theoretically consistent manner. The new theory is devoid of a major shortcoming of other similar theories; namely, the new theory enables accurate modeling of clamped boundary conditions.

Results for several example problems were presented, highlighting the superior predictive capability attainable with the present theory and its ability to model correctly clamped boundary conditions. The critical quantitative assessment of the new theory, including analyses of highly heterogeneous sandwich laminates in bending, revealed that this refined zigzag theory is more accurate than previous similar ones.

An additional and important benefit is that the new zigzag theory lends itself well to finite element approximations. In particular, the theory is perfectly suited for the development of computationally efficient, $C^{0}$-continuous finite elements. Because of a wide applicability range that includes moderately thick laminated-composite and sandwich structures, such finite elements would be highly desirable for large-scale analyses and design studies of high-performance aerospace vehicles. 


\section{Appendix: Plate stiffness coefficients}

The stiffness coefficients in (22) are computed from the following expressions using the constitutive coefficients given in (3):

- Matrix $\boldsymbol{A} \equiv\left[A_{i j}\right](i, j=1, \ldots, 3)$, symmetric, $3 \times 3$ :

$$
\boldsymbol{A} \equiv \int_{-h}^{h} \boldsymbol{C} d z
$$

- Matrix $\boldsymbol{B} \equiv\left[B_{i j}\right](i=1, \ldots, 3 ; j=1, \ldots, 7)$, nonsymmetric, $3 \times 7$ :

$$
\boldsymbol{B} \equiv \int_{-h}^{h} \boldsymbol{C} \boldsymbol{B}_{\phi} d z
$$

- Matrix $\boldsymbol{D} \equiv\left[D_{i j}\right](i, j=1, \ldots, 7)$, symmetric, $7 \times 7$ :

$$
\boldsymbol{D} \equiv \int_{-h}^{h} \boldsymbol{B}_{\phi}^{T} \boldsymbol{C} \boldsymbol{B}_{\phi} d z
$$

- Matrix $\boldsymbol{G} \equiv\left[G_{i j}\right](i, j=1, \ldots, 4)$, symmetric, $4 \times 4$ :

$$
\boldsymbol{G} \equiv \int_{-h}^{h} \boldsymbol{B}_{\beta}^{T} \boldsymbol{Q} \boldsymbol{B}_{\beta} d z
$$

where

$$
\begin{array}{rlrl}
\boldsymbol{C} & \equiv\left[\begin{array}{lll}
C_{11} & C_{12} & C_{16} \\
C_{12} & C_{22} & C_{26} \\
C_{16} & C_{26} & C_{66}
\end{array}\right]^{(k)} & \boldsymbol{B}_{\phi} \equiv\left[\begin{array}{ccccccc}
z & \phi_{1}^{(k)} & 0 & 0 & 0 & 0 & 0 \\
0 & 0 & z & \phi_{2}^{(k)} & 0 & 0 & 0 \\
0 & 0 & 0 & 0 & z & \phi_{1}^{(k)} & \phi_{2}^{(k)}
\end{array}\right], \\
\boldsymbol{Q} \equiv\left[\begin{array}{ll}
Q_{22} & Q_{12} \\
Q_{12} & Q_{11}
\end{array}\right]^{(k)}, & \boldsymbol{B}_{\beta} \equiv\left[\begin{array}{cccc}
1 & \beta_{2}^{(k)} & 0 & 0 \\
0 & 0 & 1 & \beta_{1}^{(k)}
\end{array}\right]
\end{array}
$$

\section{List of symbols}

$a, b$ Lateral dimensions of a rectangular plate.

$2 h$ Total plate (laminate) thickness.

$2 h^{(k)}$ Thickness of the $k$-th material layer (lamina).

$\left(x_{1}, x_{2}\right)$ Reference plate-coordinate axes positioned in the middle plane of the laminate.

$z$ Thickness coordinate axis.

$N$ Number of material layers (laminae) through the laminate thickness.

$S_{m}$ Reference middle plane of the laminate.

$S_{u}, S_{\sigma}$ Parts of the cylindrical edge surface of the laminate where displacements and tractions are prescribed, respectively.

$C^{0}$ Denotes a continuous function whose first-order derivative is discontinuous.

$C_{u}, C_{\sigma}$ Intersections of the cylindrical edge surfaces $\left(S_{u}, S_{\sigma}\right)$ with the middle surface $S_{m}$ where displacements and traction resultants are prescribed, respectively.

$\boldsymbol{s}, \boldsymbol{n}$ Unit outward tangential and normal vectors to the midplane boundary: see Figure 1. 
$q$ Applied transverse pressure [force/length ${ }^{2}$ ]: see Figure 1.

$\bar{T}_{1}, \bar{T}_{2}, \bar{T}_{z}$ Prescribed in-plane and transverse shear tractions: see (14).

$u_{1}^{(k)}, u_{2}^{(k)}, u_{z}$ In-plane and transverse components of the displacement vector in the $k$-th material layer: see (1).

$u, v, w$,

$\theta_{1}, \theta_{2}, \psi_{1}, \psi_{2}$ Kinematic variables of the refined zigzag plate theory: see (1).

$N_{1}, N_{2}, N_{12}$ Membrane stress resultants: see (16).

$M_{1}, M_{2}, M_{12}$ Bending and twisting stress resultants: see (17).

$M_{1}^{\phi}, M_{2}^{\phi}$ Bending stress resultants due to zigzag kinematics: see (17).

$M_{12}^{\phi}, M_{21}^{\phi}$ Twisting stress resultants due to zigzag kinematics: see (17).

$Q_{1}, Q_{2}$ Transverse shear stress resultants: see (18).

$Q_{1}^{\phi}, Q_{2}^{\phi}$ Transverse shear stress resultants due to zigzag kinematics: see (18).

$\phi_{1}^{(k)}, \phi_{2}^{(k)}$ Zigzag functions: see (1).

$\beta_{1}^{(k)}, \beta_{2}^{(k)}$ Derivatives of zigzag functions with respect to the thickness coordinate: see (2b).

$\xi^{(k)}$ Dimensionless thickness coordinates of the $k$-th layer (lamina): see (5).

$z_{(k)}$ Thickness coordinate of the interface between the $k$-th and $(k+1)$-st layers: see Figure 1.

$u_{(k)}, v_{(k)}$ Dimensionless in-plane displacements along the interface between the $k$-th and $(k+1)$-th layers: see Figure 2.

$\varepsilon_{11}^{(k)}, \varepsilon_{22}^{(k)}$ In-plane in the $k$-th layer: see (3).

$\gamma_{12}^{(k)}, \gamma_{2 z}^{(k)}, \gamma_{1 z}^{(k)}$ Transverse strains in the $k$-th layer: see (3).

$\gamma_{1}, \gamma_{2}$ Average shear strains: see (2a) and (9).

$\eta_{1}, \eta_{2}$ Transverse shear strain measures: see (11).

$\psi_{1}, \psi_{2}$ Zigzag amplitude functions: see (1).

$\sigma_{11}^{(k)}, \sigma_{22}^{(k)}$ Normal stresses in the $k$-th layer: see (3).

$\tau_{12}^{(k)}, \tau_{2 z}^{(k)}, \tau_{1 z}^{(k)}$ Transverse stresses in the $k$-th layer: see (3).

$E_{i}^{(k)}$ Young's moduli of the $k$-th layer: see Table 1.

$G_{i j}^{(k)}$ Shear moduli of the $k$-th layer: see Table 1.

$v_{i j}^{(k)}$ Poisson's ratios of the $k$-th layer: see Table 1.

$A_{i j}, B_{i j}$,

$D_{i j}, G_{i j}$ Constitutive stiffness coefficients: see (22).

$C_{i j}^{(k)}, Q_{p q}^{(k)}$ In-plane and transverse shear elastic stiffness coefficients for the $k$-th layer: see (3).

$G_{1}, G_{2}$ Weighted-average, laminate-dependent transverse shear constants: see (12b).

$\delta$ Variational operator: see (9).

$\frac{\partial}{\partial x_{\alpha}}$ or $(\cdot)_{, \alpha}$ Partial differentiation.

$k^{2}$ Shear correction factor for FSDT. 


\section{Acknowledgments}

The authors thank Dr. Scott Burton of Avago Technologies for providing the research code that was used to compute the $3 \mathrm{D}$ elasticity solutions for simply supported laminates. The first author is also very thankful to Prof. James G. Simmonds of the University of Virginia for a number of valuable technical discussions during the course of this research.

Di Sciuva and Gherlone acknowledge the Piedmont Region for the financial support of this research in the framework of Contract E57 "Multidisciplinary optimization of aeromechanical structural systems." The third author also gratefully acknowledges Politecnico di Torino for supporting his research in the framework of the Young Researchers Program (2007).

Finally, the authors thank the editorial committee of the Structural Mechanics and Concepts Branch of the NASA Langley Research Center, chaired by Dr. Michael P. Nemeth, for many valuable suggestions.

\section{References}

[Ambartsumyan 1961] S. A. Ambartsumyan, Теория анизотропных оболочек, State Publishing House for Physical and Mathematical Literature, Moscow, 1961. Translated in "Theory of anisotropic shells", NASA Technical translation TT F-118, 1964, http://tinyurl.com/NASA-TT-F-118.

[Arfken 1985] G. Arfken, Mathematical methods for physicists, 3rd ed., Academic Press, Orlando, FL, 1985.

[Averill 1994] R. C. Averill, "Static and dynamic response of moderately thick laminated beams with damage", Compos. Eng. 4:4 (1994), 381-395.

[Averill and Yip 1996] R. C. Averill and Y. C. Yip, "Development of simple, robust finite elements based on refined theories for thick laminated beams", Comput. Struct. 59:3 (1996), 529-546.

[Barut et al. 2002] A. Barut, E. Madenci, T. Anderson, and A. Tessler, "Equivalent single layer theory for a complete stress field in sandwich panels under arbitrary distributed loading", Compos. Struct. 58:4 (2002), 483-495.

[Cho and Parmerter 1992] M. Cho and R. R. Parmerter, "An efficient higher-order plate theory for laminated composites", Compos. Struct. 20:2 (1992), 113-123.

[Cho and Parmerter 1993] M. Cho and R. R. Parmerter, "Efficient higher order composite plate theory for general lamination configurations", AIAA J. 31:7 (1993), 1299-1306.

[Cook and Tessler 1998] G. M. Cook and A. Tessler, "A \{3, 2\}-order bending theory for laminated composite and sandwich beams", Compos. B Eng. 29:5 (1998), 565-576.

[Di Sciuva 1984a] M. Di Sciuva, "A refinement of the transverse shear deformation theory for multilayered orthotropic plates", in Atti del VII Congresso Nazionale AIDAA (Naples, 1983), edited by A. Marchese, ESA, Rome, 1984. Also in Aerotecnica Missili e Spazio 63 (1984), 84-92.

[Di Sciuva 1984b] M. Di Sciuva, "A refined transverse shear deformation theory for multilayered anisotropic plates", Atti Accad. Sci. Torino Cl. Sci. Fis. Mat. Natur. 118 (1984), 279-295.

[Di Sciuva 1985a] M. Di Sciuva, "Development of an anisotropic, multilayered, shear-deformable rectangular plate element", Comput. Struct. 21:4 (1985), 789-796.

[Di Sciuva 1985b] M. Di Sciuva, "Evaluation of some multilayered, shear-deformable plate elements", pp. 394-400 in A collection of technical papers: AIAA/ASME/ASCE/AHS 26th Structures, Structural Dynamics and Materials Conference (Orlando, FL, 1985), vol. 1, AIAA, New York, 1985. Paper 85-0717.

[Di Sciuva 1986] M. Di Sciuva, "Bending, vibration and buckling of simply supported thick multilayered orthotropic plates: an evaluation of a new displacement model", J. Sound Vib. 105:3 (1986), 425-442.

[Di Sciuva 1987] M. Di Sciuva, "An improved shear-deformation theory for moderately thick multilayered anisotropic shells and plates", J. Appl. Mech. (ASME) 54:3 (1987), 589-596.

[Di Sciuva 1990] M. Di Sciuva, "Further refinement in the transverse shear deformation theory for multilayered composite plates", Atti Accad. Sci. Torino Cl. Sci. Fis. Mat. Natur. 124:5-6 (1990), 248-268. 
[Di Sciuva 1992] M. Di Sciuva, "Multilayered anisotropic plate models with continuous interlaminar stresses", Compos. Struct. 22:3 (1992), 149-168.

[Di Sciuva et al. 2002] M. Di Sciuva, M. Gherlone, and L. Librescu, "Implications of damaged interfaces and of other nonclassical effects on the load carrying capacity of multilayered composite shallow shells", Int. J. Non-Linear Mech. 37:4-5 (2002), 851-867.

[Kim and Cho 2005] J.-S. Kim and M. Cho, "Enhanced first-order shear deformation theory for laminated and sandwich plates", J. Appl. Mech. (ASME) 72:6 (2005), 809-817.

[Kim and Cho 2006] J.-S. Kim and M. Cho, "Enhanced modeling of laminated and sandwich plates via strain energy transformation”, Compos. Sci. Technol. 66:11-12 (2006), 1575-1587.

[Librescu et al. 1987] L. Librescu, A. A. Khdeir, and J. N. Reddy, "A comprehensive analysis of the state of stress of elastic anisotropic flat plates using refined theories", Acta Mech. 70:1-4 (1987), 57-81.

[Liu and Li 1996] D. Liu and X. Li, "An overall view of laminate theories based on displacement hypothesis", J. Compos. Mater. 30:14 (1996), 1539-1561.

[Lo et al. 1977a] K. H. Lo, R. M. Christensen, and E. M. Wu, "A higher-order theory of plate deformation, 1: Homogeneous plates", J. Appl. Mech. (ASME) 44 (1977), 663-668.

[Lo et al. 1977b] K. H. Lo, R. M. Christensen, and E. M. Wu, "A higher-order theory of plate deformation, 2: Laminated plates", J. Appl. Mech. (ASME) 44 (1977), 669-676.

[MSC 2006] MD Nastran: reference guide, Version 2006.0, MSC Software Corporation, Santa Ana, CA, 2006.

[Murakami 1986] H. Murakami, "Laminated composite plate theory with improved in-plane responses", J. Appl. Mech. (ASME) 53:3 (1986), 661-666.

[Noor and Burton 1989] A. K. Noor and W. S. Burton, "Assessment of shear deformable theories for multilayered composite plates", Appl. Mech. Rev. (ASME) 42:1 (1989), 1-12.

[Noor and Burton 1990] A. K. Noor and W. S. Burton, "Three-dimensional solutions for antisymmetrically laminated anisotropic plates", J. Appl. Mech. (ASME) 57:1 (1990), 182-188.

[Pagano 1969] N. J. Pagano, "Exact solutions for composite laminates in cylindrical bending”, J. Compos. Mater. 3:3 (1969), 398-411.

[Reddy 1984] J. N. Reddy, “A simple higher-order theory for laminated composite plates", J. Appl. Mech. (ASME) 51:4 (1984), 745-752.

[Reddy 1997] J. N. Reddy, Mechanics of laminated composite plates: theory and analysis, CRC Press, Boca Raton, FL, 1997.

[Reddy 2004] J. N. Reddy, Mechanics of laminated composite plates and shells: theory and analysis, 2nd ed., CRC Press, Boca Raton, FL, 2004.

[Reddy and Vijayakumar 1995] K. J. Reddy and K. Vijayakumar, "Lamination-dependent shear deformation models for cylindrical bending of angle-ply laminates", Comput. Struct. 55:4 (1995), 717-725.

[Reissner 1984] E. Reissner, "On a certain mixed variational theorem and a proposed application", Int. J. Numer. Methods Eng. 20:7 (1984), 1366-1368.

[Reissner 1985] E. Reissner, "Reflections on the theory of elastic plates", Appl. Mech. Rev. (ASME) 38:11 (1985), 1453-1464.

[Sun and Whitney 1973] C.-T. Sun and J. M. Whitney, "Theories for the dynamic response of laminated plates", AIAA J. 11:2 (1973), 178-183.

[Tessler 1993] A. Tessler, "An improved plate theory of $\{1,2\}$-order for thick composite laminates", Int. J. Solids Struct. 30:7 (1993), 981-1000.

[Tessler et al. 2007] A. Tessler, M. Di Sciuva, and M. Gherlone, "Refinement of Timoshenko beam theory for composite and sandwich beams using zigzag kinematics", Technical report TP-2007-215086, NASA Langley Research Center, Hampton, VA, 2007, Available at http://tinyurl.com/NASA-TP-2007-215086.

[Tessler et al. 2009] A. Tessler, M. Di Sciuva, and M. Gherlone, "Refined zigzag theory for laminated composite and sandwich plates", Technical report TP-2009-215561, NASA Langley Research Center, Hampton, VA, 2009, Available at http:// tinyurl.com/NASA-TP-2009-215561. 
[Toledano and Murakami 1987] A. Toledano and H. Murakami, "A high-order laminated plate theory with improved in-plane responses", Int. J. Solids Struct. 23:1 (1987), 111-131.

[Umasree and Bhaskar 2006] P. Umasree and K. Bhaskar, "Analytical solutions for flexure of clamped rectangular cross-ply plates using an accurate zig-zag type higher-order theory", Compos. Struct. 74:4 (2006), 426-439.

[Vlachoutsis 1992] S. Vlachoutsis, "Shear correction factors for plates and shells", Int. J. Numer. Methods Eng. 33:7 (1992), $1537-1552$.

[Yu 2005] W. Yu, "Mathematical construction of a Reissner-Mindlin plate theory for composite laminates", Int. J. Solids Struct. 42:26 (2005), 6680-6699.

[Yu et al. 2008] W. Yu, J.-S. Kim, D. H. Hodges, and M. Cho, "A critical evaluation of two Reissner-Mindlin type models for composite laminated plates", Aerosp. Sci. Technol. 12:5 (2008), 408-417.

Received 23 Mar 2009. Revised 7 Aug 2009. Accepted 13 Aug 2009.

ALEXANDER TESSLER: alexander.tessler-1@nasa.gov

Structural Mechanics and Concepts Branch, NASA Langley Research Center, Mail Stop 190, Hampton, VA 23681-2199, United States

MARCo Di ScIUVA: marco.disciuva@polito.it

Department of Aeronautics and Space Engineering, Politecnico di Torino, Corso Duca degli Abruzzi 24, 10129 Torino, Italy

MARCo GHERLONE: marco.gherlone@polito.it

Department of Aeronautics and Space Engineering, Politecnico di Torino, Corso Duca degli Abruzzi 24, 10129 Torino, Italy 


\title{
JOURNAL OF MECHANICS OF MATERIALS AND STRUCTURES
}

\author{
http://www.jomms.org
}

\author{
Founded by Charles R. Steele and Marie-Louise Steele

\section{EDITORS} \\ Charles R. STEele \\ DAVIDE BIGONI \\ IWONA JASIUK \\ YASUHIDE SHINDO \\ Stanford University, U.S.A. \\ University of Trento, Italy \\ University of Illinois at Urbana-Champaign, U.S.A. \\ Tohoku University, Japan
}

\section{EDITORIAL BOARD}

H. D. BUI École Polytechnique, France

J. P. CARTER University of Sydney, Australia

R. M. Christensen Stanford University, U.S.A.

G. M. L. GLADWELL University of Waterloo, Canada

D. H. HodGES Georgia Institute of Technology, U.S.A.

J. HUTCHINSON Harvard University, U.S.A.

C. HwU National Cheng Kung University, R.O. China

B. L. KariHaloo University of Wales, U.K.

Y. Y. KIM Seoul National University, Republic of Korea

Z. Mroz Academy of Science, Poland

D. PAMPlonA Universidade Católica do Rio de Janeiro, Brazil

M. B. RUBIN Technion, Haifa, Israel

A. N. SHUPIKov Ukrainian Academy of Sciences, Ukraine

T. TARNAI University Budapest, Hungary

F. Y. M. WAN University of California, Irvine, U.S.A.

P. WRIGGERS Universität Hannover, Germany

W. YANG Tsinghua University, P.R. China

F. ZIEGLER Technische Universität Wien, Austria

\section{PRODUCTION}

\section{Paulo Ney de Souza Production Manager \\ SheIla Newbery Senior Production Editor \\ SILVIO LEVY Scientific Editor}

See inside back cover or http://www.jomms.org for submission guidelines.

JoMMS (ISSN 1559-3959) is published in 10 issues a year. The subscription price for 2010 is US $\$ 500 /$ year for the electronic version, and \$660/year (+\$60 shipping outside the US) for print and electronic. Subscriptions, requests for back issues, and changes of address should be sent to Mathematical Sciences Publishers, Department of Mathematics, University of California, Berkeley, CA 94720-3840.

JoMMS peer-review and production is managed by EditFLOW ${ }^{\mathrm{TM}}$ from Mathematical Sciences Publishers.

PUBLISHED BY

mathematical sciences publishers

http://www.mathscipub.org

A NON-PROFIT CORPORATION

Typeset in LATEX

CCopyright 2010. Journal of Mechanics of Materials and Structures. All rights reserved. 


\title{
Journal of Mechanics of Materials and Structures
}

\author{
Volume 5, No. 2 February 2010
}

A critical analysis of interface constitutive models for the simulation of delamination in composites and failure of adhesive bonds

Anton Matzenmiller, Sebastian Gerlach and Mark Fiolka

185

Computational studies of collagen fibril biominerals using a virtual internal bond

model with extrinsic length scale

Ganesh Thiagarajan and Kavita Deshmukh

The simulation of stochastically excited viscoelastic systems and their stability

VADIM D. POTAPOV

Fundamental solutions for an inhomogeneous cross-anisotropic material due to horizontal and vertical plane strain line loads

Cheng-Der Wang, Jia-YAN Hou and WeI-Jer WANG

Mechanical and fracture analysis of welded pearlitic rail steels

Aldinton Allie, Heshmat A. Aglan and Mahmood Fateh

Rate dependence of indentation size effects in filled silicone rubber

Ramanjaneyulu V. S. TATIRAJU and ChUng-SOUK HAN

A novel application of a laser Doppler vibrometer in a hèalth monitoring system

DAVOOD REZAEI and FARID TAHERI

Energy absorption of a helicoidal bistable structure

Seubpong Leelavanichkul, Andrej Cherkaev, Daniel O. Adams

and FLORIAN SOLZBACHER

Decay properties of solutions of a Mindlin-type plate model for rhombic systems

Francesca Passarella, Vincenzo Tibullo and VitTorio Zampoli

A consistent refinement of first-order shear deformation theory for laminated composite and sandwich plates using improved zigzag kinematics Alexander Tessler, Marco Di Sciuva and Marco Gherlone 